(c) 2006 International Press

Adv. Theor. Math. Phys. 10 (2006) 879-913

\title{
On matrix differential equations in the Hopf algebra of renormalization
}

\author{
Kurusch Ebrahimi-Fard ${ }^{1}$ and Dominique Manchon ${ }^{2}$ \\ ${ }^{1}$ I.H.É.S., Le Bois-Marie, 35, Rt de Chartres, \\ F-91440 Bures-sur-Yvette, France \\ kurusch@ihes.fr \\ ${ }^{2}$ Université Blaise Pascal, C.N.R.S.-UMR 6620, \\ 63177 Aubière, France \\ manchon@math.univ-bpclermont.fr
}

\begin{abstract}
We establish Sakakibara's differential equations [M. Sakakibara, On the differential equations of the characters for the renormalization group, Mod. Phys. Lett. A 19 (2004), 1453.] in a matrix setting for the counter term (respectively renormalized character) in Connes-Kreimer's Birkhoff decomposition in any connected graded Hopf algebra, thus including Feynman rules in perturbative renormalization as a key example.
\end{abstract}

\section{Introduction}

Quantum field theory (QFT) unifies the fundamental principles of special relativity and quantum theory and provides the appropriate physical

e-print archive: http://lanl.arXiv.org/abs/math-ph/0606039 
framework to describe phenomena at the smallest length scales respectively highest energies. Its mathematical structure is far from being as simple as that of its basic constituents. Moreover, up to now, perturbation theory is the most successful quantitative and qualitative approach to QFT. Although general agreement between theoretically predicted results in the perturbative regime of QFT and those experimentally measured has reached a satisfactory status, a truly non-perturbative understanding of the physics of quantum phenomena is mandatory, both for future advancements in terms of fundamental as well as calculational problems.

Perturbative QFT consist of two fundamental ingredients, the gauge principle and the concept of renormalization. The latter consists of an arbitrary regularization prescription, which parameterizes ultraviolet divergencies appearing in Feynman amplitudes and thereby renders them formally finite, together with a specific subtraction rule of those ill-defined expressions dictated by physical principles. Whereas both the gauge principle and the concept of renormalization experienced a rich development in theoretical physics, the former especially came to the fore of mathematical research with rich interactions between mathematicians and physicists. However, the latter suffered from the lack of an equally strong development of its mathematical aspects.

Kreimer's recent findings [31,32] mark a turning point in this context. He discovered a mathematical structure underlying renormalization in perturbative QFT in terms of connected graded commutative Hopf algebras. Feynman rules are interpreted as Hopf algebra characters which associate to each Feynman graph its corresponding amplitude.

The concept of regularization in general introduces non-physical parameters. This process changes the nature of Feynman rules drastically, i.e., from linear multiplicative maps into the underlying base field to algebra morphisms with image in a commutative unital algebra, e.g., Laurent series in dimensional regularization. Hence, we identify regularized Feynman rules with a particular subclass of such maps from the Hopf algebra of Feynman graphs into a commutative unital algebra dictated by the regularization scheme.

Connes and Kreimer extended the results on the Hopf algebraic approach to perturbative renormalization by establishing the Hopf algebra of Feynman graphs including the concept of the renormalization group $[9,10,11,12]$. Moreover, Connes and Kreimer formulated in this picture the intricate process of perturbative renormalization in terms of an algebraic Birkhoff decomposition of regularized Feynman rules, using the minimal subtraction scheme in dimensional regularization. 
In $[19,20]$, it was shown how to organize the combinatorics of renormalization in terms of (pro-)nil- and unipotent triangular matrix representations with entries in a commutative Rota-Baxter algebra. A simple factorization of such matrices was derived using explicit non-recursive equations containing the renormalization scheme operator. This simple matrix decomposition offers a transparent picture of the process of renormalization in terms of the factorization of Feynman rules matrices.

In this work, we would like to further develop the matrix calculus approach to perturbative renormalization in the abstract context of connected graded Hopf algebras. Any left coideal gives rise to a representation of the group of characters of the Hopf algebra by lower triangular unipotent matrices, the size of which being given by the dimension of the coideal. We investigate the matrix representation of two fundamental concepts which can be defined in this purely algebraic framework: the renormalization group and the beta-function. We retrieve then M. Sakakibara's differential equations involving the beta-function, giving to his approach the firm ground of triangular matrix calculus.

Before starting, we should point the reader to the following papers $[6,14,16,22,24,25,35,36,39,50]$, and books $[13,26,27,34,40,51]$, which are useful as introductory references, both with respect to perturbative QFT and renormalization theory, as well as its recently discovered Hopf-algebraic structures. Also, some readers may find it stimulating to leaf through the books by Brown [5] and Schweber [44] as well as the more recent one by Kaiser [29] for some scientific-historical perspectives on Feynman graphs in QFT and renormalization theory. Schwinger's collection of reprints [45] contains many of the original articles marking the beginning of modern perturbative QFT and renormalization theory. Comprehensive treatments of Hopf algebras can be found in $[1,49]$, see also the paper by Bergman [3]. Other useful references are $[7,26,30,38,48]$. Hopf algebras in the context of combinatorics appeared in the work of Rota [42], and Joni and Rota [28], see also $[25,41,46,47]$.

Let us briefly outline the organization of this paper. In Section 2, after reminding Connes-Kreimer's Birkhoff decomposition of characters in the most general context of connected filtered Hopf algebras, we define the matrix representation associated with a left coideal, along the lines of [20], and write down the matrix counterpart of the Birkhoff decomposition. In Section 3, we first define the renormalization group and the beta-function in the context of connected graded Hopf algebras, along the lines of [10] and [39], and then we describe the matrix counterparts of these notions. The key point is that the grading biderivation $Y$ of the Hopf algebra can be represented by a diagonal matrix. The semidirect product of the group 
of characters with the associated one-parameter group of automorphisms can then be represented by (non-unipotent) lower triangular matrices. The two last subsections are devoted to a careful rewriting of some important results of M. Sakakibara [43] in the matrix representation, yielding matrix differential equations for the beta-function.

\section{The general set up}

In the sequel, $k$ denotes the ground field with $\operatorname{char}(k)=0$ over which all algebraic structures are defined. Here the term algebra always means unital associative $k$-algebra, denoted by the triple $\left(\mathcal{A}, m_{\mathcal{A}}, \eta_{\mathcal{A}}\right)$, where $\mathcal{A}$ is a $k$ vector space with a product $m_{\mathcal{A}}: \mathcal{A} \otimes \mathcal{A} \rightarrow \mathcal{A}$ and a unit map $\eta_{\mathcal{A}}: k \rightarrow \mathcal{A}$. Similarly, for coalgebras over $k$, denoted by the triple $\left(\mathcal{C}, \Delta_{\mathcal{C}}, \epsilon_{\mathcal{C}}\right)$, where the coproduct map $\Delta_{\mathcal{C}}: \mathcal{C} \rightarrow \mathcal{C} \otimes \mathcal{C}$ is coassociative and $\epsilon_{\mathcal{C}}: \mathcal{C} \rightarrow k$ denotes the counit map. A subspace $\mathcal{J} \subset \mathcal{C}$ is called a left coideal if $\Delta_{\mathcal{C}}(\mathcal{J}) \subset \mathcal{C} \otimes \mathcal{J}$. A Hopf algebra, denoted by $\left(\mathcal{H}, m_{\mathcal{H}}, \eta_{\mathcal{H}}, \Delta_{\mathcal{H}}, \epsilon_{\mathcal{H}}, S\right)$, is a bialgebra together with the antipode $S: \mathcal{H} \rightarrow \mathcal{H}$, that is, it consists of an algebra and coalgebra structure in a compatible way and $S$ is a $k$-linear map on $\mathcal{H}$ satisfying the Hopf algebra axioms [1,49]. In the following, we omit subscripts for notational transparency if there is no danger of confusion and denote algebras, coalgebras and Hopf algebras simply by $\mathcal{A}, \mathcal{C}$ and $\mathcal{H}$, respectively.

\subsection{Connected filtered Hopf algebra}

Let $\mathcal{H}$ be a connected filtered bialgebra:

$$
k=\mathcal{H}^{(0)} \subset \mathcal{H}^{(1)} \subset \cdots \subset \mathcal{H}^{(n)} \subset \cdots, \quad \bigcup_{n \geq 0} \mathcal{H}^{(n)}=\mathcal{H},
$$

and let $\mathcal{A}$ be any commutative algebra. The space $\mathcal{L}(\mathcal{H}, \mathcal{A})$ of linear maps from $\mathcal{H}$ to $\mathcal{A}$ together with the convolution product $f \star g:=m_{\mathcal{A}} \circ(f \otimes g) \circ$ $\Delta, f, g \in \mathcal{L}:$

$$
\mathcal{H} \stackrel{\Delta}{\longrightarrow} \mathcal{H} \otimes \mathcal{H} \stackrel{f \otimes g}{\longrightarrow} \mathcal{A} \otimes \mathcal{A} \stackrel{m_{\mathcal{A}}}{\longrightarrow} \mathcal{A}
$$

is an algebra with unit $e:=\eta_{\mathcal{A}} \circ \epsilon$. For any $x \in \mathcal{H}^{(n)}$, we have, using a variant of Sweedler's notation [49],

$$
\Delta(x)=x \otimes 1+1 \otimes x+\sum_{(x)} x^{\prime} \otimes x^{\prime \prime},
$$

where the filtration degrees of $x^{\prime}$ and $x^{\prime \prime}$ are strictly smaller than $n$. Recall that by definition we call an element $x \in \mathcal{H}$ primitive if

$$
\bar{\Delta}(x):=\Delta(x)-x \otimes 1-1 \otimes x=0 .
$$


The convolution product on $\mathcal{L}(\mathcal{H}, \mathcal{A})$ writes then with Sweedler's notation:

$$
(f \star g)(x)=f(x) g(1)+f(1) g(x)+\sum_{(x)} f\left(x^{\prime}\right) g\left(x^{\prime \prime}\right) \in \mathcal{A} .
$$

The filtration of $\mathcal{H}$ implies a decreasing filtration on $\mathcal{L}(\mathcal{H}, \mathcal{A})$ in terms of $\mathcal{L}^{n}:=\left\{f \in \mathcal{L} \mid f_{\left.\right|_{\mathcal{H}^{(n-1)}}}=0\right\}$ and $\mathcal{L}(\mathcal{H}, \mathcal{A})$ is complete with respect to the induced topology [39]. The subset $\mathfrak{g}_{0}:=\mathcal{L}^{1} \subset \mathcal{L}(\mathcal{H}, \mathcal{A})$ of linear maps $\alpha$ that send the bialgebra unit to zero, $\alpha(1)=0$, forms a Lie algebra in $\mathcal{L}(\mathcal{H}, \mathcal{A})$. The exponential

$$
\exp ^{\star}(\alpha)=\sum_{k} \frac{1}{k !} \alpha^{\star k}
$$

makes sense and is a bijection from $\mathfrak{g}_{0}$ onto the group $G_{0}=e+\mathfrak{g}_{0}$ of linear maps $\gamma$ that send the bialgebra unit to the algebra unit, $\alpha(1)=1_{\mathcal{A}}[39]$. Here and further below, the particular notation of the exponential map, $\exp ^{\star}$, indicates its definition with respect to the convolution product (2.2). Hence, on the right-hand side $\alpha^{\star k}$ denotes the $k$-fold convolution product of $\alpha$ with itself.

An infinitesimal character with values in $\mathcal{A}$ is a linear map $\xi \in \mathcal{L}(\mathcal{H}, \mathcal{A})$ such that for $x, y \in \mathcal{H}$,

$$
\xi(x y)=\xi(x) e(y)+e(x) \xi(y) .
$$

We denote by $\mathfrak{g}_{\mathcal{A}} \subset \mathfrak{g}_{0}$ the linear space of infinitesimal characters. We call an $\mathcal{A}$-valued map $\rho$ in $\mathcal{L}(\mathcal{H}, \mathcal{A})$ a character if $\rho(1)=1$ and for $x, y \in \mathcal{H}$

$$
\rho(x y)=\rho(x) \rho(y) .
$$

The set of such unital algebra morphisms is denoted by $G_{\mathcal{A}} \subset G_{0}$.

Let us now assume that $\mathcal{A}$ is a commutative algebra. It is easily verified then, see for instance [39], that the set $G_{\mathcal{A}}$ of characters from $\mathcal{H}$ to $\mathcal{A}$ forms a group for the convolution product. In fact it is the pro-unipotent group ${ }^{1}$ of $\mathcal{A}$-valued morphisms on the bialgebra $\mathcal{H}$, and $\mathfrak{g}_{\mathcal{A}}$ in $\mathfrak{g}_{0}$ is the corresponding pro-nilpotent Lie algebra. The exponential map, $\exp ^{\star}$, restricts to a bijection between $\mathfrak{g}_{\mathcal{A}}$ and $G_{\mathcal{A}}$. The neutral element $e:=\eta_{\mathcal{A}} \circ \epsilon$ in $G_{\mathcal{A}}$ is given by $e(1)=1_{\mathcal{A}}$ and $e(x)=0$ for $x \in \operatorname{Ker} \epsilon$. The inverse of $\varphi \in G_{\mathcal{A}}$ is given by composition with the antipode $S$ :

$$
\varphi^{\star-1}=\varphi \circ S
$$

\footnotetext{
${ }^{1}$ It is more precisely a group scheme, i.e., a functor $\mathcal{A} \mapsto G_{\mathcal{A}}$ from $k$-algebras to groups.
} 
Here, for the reader's convenience, we emphasize that the notation $\varphi^{\star-1}$ means the inverse with respect to the convolution product (2.2).

Recall that the antipode $S: \mathcal{H} \rightarrow \mathcal{H}$ is the inverse of the identity for the convolution product on $\mathcal{L}(\mathcal{H}, \mathcal{H})$ :

$$
S \star I d=m \circ(S \otimes I d) \circ \Delta=\eta \circ \epsilon=I d \star S .
$$

It always exists in a connected filtered bialgebra, hence any connected filtered bialgebra is a connected filtered Hopf algebra. The antipode is defined by

$$
S=\sum_{n \geq 0}(\eta \circ \epsilon-I d)^{\star n}
$$

Recall that $\Delta^{(0)}:=I d$ and for $n>0 \Delta^{(n)}:=\left(\Delta^{(n-1)} \otimes I d\right) \circ \Delta$. Equations (2.6) imply the following recursive formulas for the antipode starting with $S(1)=1$ and for $x \in \operatorname{Ker} \epsilon$ :

$$
\begin{aligned}
& S(x)=-x-\sum_{(x)} S\left(x^{\prime}\right) x^{\prime \prime} \\
& S(x)=-x-\sum_{(x)} x^{\prime} S\left(x^{\prime \prime}\right) .
\end{aligned}
$$

Example 2.1 (Toy-model of decorated non-planar rooted trees). As a guiding example, we will use the Hopf algebra of non-planar rooted trees established by Kreimer [32]. It provides the role model for the Hopf algebraic formulation of perturbative renormalization [9]. In fact, linear combinations of - decorated - non-planar rooted trees naturally encode the hierarchical structure of divergencies of a Feynman graph. Each vertex of a rooted tree represents a primitive divergence indicated by a decoration with a primitive one-particle irreducible (1PI) Feynman graph. Edges connecting such vertices encode thereby the nesting of subdivergencies, i.e., proper 1PI subgraphs sitting inside another 1PI graph. The root vertex is the overall divergence which contains those subdivergencies. Linear combinations of such decorated rooted trees may represent Feynman graphs with overlapping divergence structures [33].

By definition, a rooted tree $t$ is made out of vertices and non-intersecting oriented edges, such that all but one vertex have exactly one incoming line. We denote the set of vertices and edges of a rooted tree by $V(t), E(t)$, respectively. The root is the only vertex with no incoming line. We draw the root on top of the tree. Let $T$ denote the set of isomorphic classes of 
rooted trees. The empty tree is denoted by $1_{\mathcal{T}}$ :

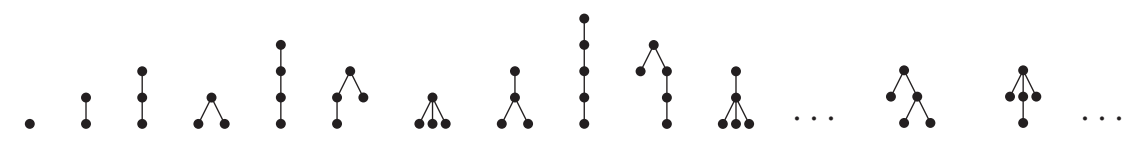

Let $\mathcal{T}$ be the $k$-vector space generated by $T$, which is graded by the number of vertices, $\operatorname{deg}(t):=|V(t)|, t \in \mathcal{T}$, with the convention that $\operatorname{deg}\left(1_{\mathcal{T}}\right)=0$. Later, for the sake of notational transparency, we denote the degree of a tree $t \in \mathcal{T}$ by $|t|:=\operatorname{deg}(t)$. Let $\mathcal{H}_{\mathcal{T}}$ be the graded commutative polynomial algebra of finite type over $k$ generated by $\mathcal{T}, \mathcal{H}_{\mathcal{T}}:=k[\mathcal{T}]=\bigoplus_{n>0} \mathcal{H}^{(n)}$. Monomials of trees are called forests. We extend $\operatorname{deg}\left(t_{1} \ldots t_{n}\right):=\sum_{i=1}^{n} \operatorname{deg}\left(t_{i}\right)$.

We will define a coalgebra structure on $\mathcal{H}_{\mathcal{T}}$. The coproduct is defined in terms of cuts $c \subset E(t)$ on a tree $t \in \mathcal{T}$. A primitive cut is the removal of a single edge, $|c|=1$, from the tree $t$. The tree $t$ decomposes into two parts, denoted by the pruned part $P_{c}(t)$ and the rooted part $R_{c}(t)$, where the latter contains the original root vertex. An admissible cut of a rooted tree $t$ is a set of primitive cuts, $|c| \geq 1$, such that along the unique path from the root to any vertex of $t$, one encounters at most one cut.

Let $C_{t}$ be the set of all admissible cuts of the rooted tree $t \in \mathcal{T}$. We exclude the empty cut $c^{(0)}: P_{c^{(0)}}(t)=\emptyset, R_{c^{(0)}}(t)=t$, and the full cut $c^{(1)}$ : $P_{c^{(1)}}(t)=t, R_{c^{(1)}}(t)=\emptyset$. Also let $C_{t}^{(01)}$ be $C_{t} \cup\left\{c^{(0)}(t), c^{(1)}(t)\right\}$. The coproduct is defined by

$$
\Delta(t):=t \otimes 1_{\mathcal{T}}+1_{\mathcal{T}} \otimes t+\sum_{c \in C_{t}} P_{c}(t) \otimes R_{c}(t)=\sum_{c \in C_{t}^{(01)}} P_{c}(t) \otimes R_{c}(t)
$$

We shall call the rooted tree $R_{c}(t)$ the cotree (or cograph) corresponding to the admissible cut $c$ on the tree $t$. One sees easily that $\operatorname{deg}(t)=\operatorname{deg}\left(P_{c}(t)\right)+$ $\operatorname{deg}\left(R_{c}(t)\right)$, for all admissible cuts $c \in C_{t}$, and therefore

$$
\bar{\Delta}(t)=\sum_{c \in C_{t}} P_{c}(t) \otimes R_{c}(t) \in \sum_{p+q=\operatorname{deg}(t), p, q>0} \mathcal{H}^{(p)} \otimes \mathcal{H}^{(q)}
$$

Furthermore, this map is extended by definition to an algebra morphism on $\mathcal{H}_{\mathcal{T}}$ :

$$
\Delta\left(\prod_{i=1}^{n} t_{i}\right):=\prod_{i=1}^{n} \Delta\left(t_{i}\right)
$$


The best way to get use to this particular coproduct is to present some examples:

$$
\begin{aligned}
& \Delta(\bullet)=\bullet 1_{\mathcal{T}}+1_{\mathcal{T}} \otimes \bullet \\
& \Delta(\mathfrak{\bullet})=\mathfrak{\imath} \otimes 1_{\mathcal{T}}+1_{\mathcal{T}} \otimes \mathfrak{\bullet}+\bullet \otimes \bullet \\
& \Delta(\bullet)=\Delta(\bullet) \Delta(\bullet)=\bullet \otimes 1_{\mathcal{T}}+1_{\mathcal{T}} \otimes \bullet \bullet+2 \bullet \bullet \\
& \Delta(\diamond)=\AA \otimes 1_{\mathcal{T}}+1_{\mathcal{T}} \otimes \AA+2 \bullet \otimes \mathfrak{\bullet}+\bullet \otimes \bullet \\
& \Delta(\cdot \mathfrak{l})=\Delta(\mathfrak{\bullet})=\Delta(\bullet) \Delta(\mathfrak{l}) \\
& =\bullet \otimes 1_{\mathcal{T}}+1_{\mathcal{T}} \otimes \bullet \bullet+\bullet \otimes \bullet+\bullet \otimes \bullet \cdot+\bullet \otimes \bullet+\bullet \otimes:
\end{aligned}
$$

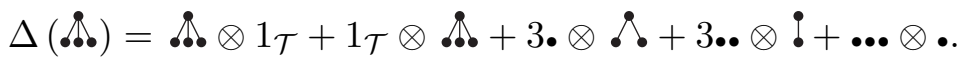

One observes immediately that the vector space $\mathcal{T}$ defines a left coideal, i.e.,

$$
\mathcal{T} \stackrel{\Delta}{\longrightarrow} \mathcal{H}_{\mathcal{T}} \otimes \mathcal{T}
$$

The experienced reader will easily recognize that this coproduct efficiently stores the so-called wood $\mathfrak{W}(\Gamma)$ for the Feynman graph $\Gamma$ with its hierarchy of subdivergencies represented by the tree $t \sim \Gamma$. A wood simply contains all spinneys $^{2}$ of the graph $[6,21]$. The right-hand side of the above coproduct consists of the cograph following from the contraction of the corresponding spinney denoted on the left-hand side.

Connes and Kreimer showed that $\mathcal{H}_{\mathcal{T}}$ with coproduct (2.10), and counit $\epsilon$ defined by $\epsilon(1):=1_{k}$ and zero else is a connected $\mathbb{Z}_{\geq 0}$-graded commutative, non-cocommutative bialgebra of finite type and hence a Hopf algebra, with antipode $S$ defined recursively by $S\left(1_{\mathcal{T}}\right)=1_{\mathcal{T}}$ and

$$
S(t):=-t-\sum_{c \in C_{t}} S\left(P_{c}(t)\right) R_{c}(t) .
$$

Again, a couple of examples might be helpful here:

$$
\begin{aligned}
& S(\bullet)=-\bullet \\
& S(\mathfrak{\bullet})=-\mathfrak{\bullet}-S(\bullet) \cdot=-\mathfrak{\bullet}+\bullet \\
& S(\AA)=-\AA-2 S(\bullet) \bullet-S(\bullet) \bullet=-\AA+2 \bullet \bullet-\bullet \bullet \\
& S(\AA)=-\AA-3 S(\bullet) \wedge-3 S(\bullet) \bullet-S(\bullet \bullet) \bullet \\
& =-\AA+3 . \AA-3 . . \bullet+\cdots .
\end{aligned}
$$

\footnotetext{
${ }^{2} \mathrm{~A}$ spinney is a - possibly non-connected — subgraph with one-particle irreducible components.
} 
We chose a simple decoration of tree vertices by tree factorials $[32,37]$ defined as follows. Let $t \in \mathcal{T}$, each primitive cut, that is, each edge $c \in E(t)$, defines two rooted trees, i.e., the pruned tree $t_{v_{c}}:=P_{c}(t)$ and $R_{c}(t)$. The root of the former is the vertex $v_{c} \in V(t)$ which had $c$ as its incoming edge. The root of the cotree $R_{c}(t)$ is the original root. Obviously, $\operatorname{deg}(t)=\operatorname{deg}\left(t_{v_{c}}\right)+$ $\operatorname{deg}\left(R_{c}(t)\right)$. The tree-factorial of $t$ is defined by

$$
t^{!}:=\prod_{\substack{c \in C_{t}^{(1)} \\|c|=1}} \operatorname{deg}\left(t_{v_{c}}\right)=\prod_{v \in V(t)} \operatorname{deg}\left(t_{v}\right)
$$

The second equality is clear as to each vertex we can associate the unique incoming edge $c \in E(t)$. As examples, we mention

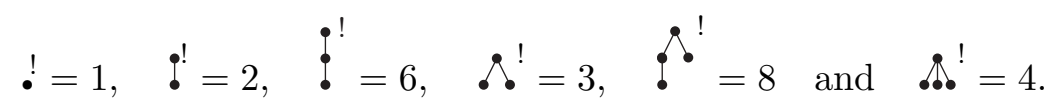

In the following, we decorate each vertex $v \in V(t)$ of a rooted tree $t \in \mathcal{T}$ by its tree factorial, $t_{v}^{!}$. For notational transparency, we omit the decorations on the trees.

Recall the function

$$
\int_{0}^{\infty} y^{-a z}(y+c)^{-1-b z} d y=B((a+b) z, 1-a z) c^{-(a+b) z},
$$

where

$$
B(u, v):=\frac{\Gamma(u) \Gamma(v)}{\Gamma(u+v)}
$$

and $c>0$. Here $\Gamma(a)$ is the usual Euler Gamma-function [34]. We define now the family of functions:

$$
B_{n}:=B_{n}(z):=B(n z, 1-n z), \quad n>0 .
$$

Following [32,37], we define the following regularized toy-model character $\varphi=\varphi(a, \mu, z)$ from the Hopf algebra of integer decorated rooted trees $\mathcal{H}_{\mathcal{T}}$ to $\left.\mathcal{A}:=\mathbb{C}\left[z^{-1}, z\right]\right][[\log (a / \mu)]], a / \mu>0: \varphi\left(1_{\mathcal{T}}\right)(a, \mu, z):=1_{\mathcal{A}}$,

$$
\varphi(t)(a, \mu, z):=\left(\frac{a}{\mu}\right)^{-z \operatorname{deg}(t)} \prod_{v \in V(t)} B_{w\left(t_{v}\right)}, \quad \text { for } t \in \operatorname{Ker} \epsilon .
$$

Here, $a$ is assumed to be a dimensional external parameter, and $\mu$ is the so-called 't Hooft mass or renormalization scale. The latter is an arbitrarily chosen parameter of the same dimension as $a$, such that the ratio $a / \mu$ is a positive number. The parameter $\mu$ introduces an external scale specific 
to dimensional regularization [13]. Let us give a few examples by applying $\varphi(a, \mu, z)$ to some trees. Defining $\alpha:=a / \mu$, we find

$$
\begin{aligned}
\varphi(\bullet)(a, \mu, z) & =\alpha^{-z} B_{1}=\alpha^{-z} B(z, 1-z) \\
& =\alpha^{-z} \frac{\pi}{\sin \pi z}=\alpha^{-z}\left(\frac{1}{z}+\frac{\pi^{2}}{6} z+O\left(z^{2}\right)\right) \\
\varphi(\bullet)(a, \mu, z) & =\alpha^{-2 z} B_{2} B_{1} \\
& =\alpha^{-2 z} B(2 z, 1-2 z) B(z, 1-z), \\
\varphi(\AA)(a, \mu, z) & =\alpha^{-3 z} B_{3} B_{1}^{2} \\
& =\alpha^{-3 z} B(3 z, 1-3 z) B(z, 1-z) B(z, 1-z), \\
\varphi(\AA)(a, \mu, z) & =\alpha^{-4 z} B_{4} B_{1}^{3} \\
& =\alpha^{-4 z} B(4 z, 1-4 z) B(z, 1-z) B(z, 1-z) B(z, 1-z), \\
\varphi(\AA)(a, \mu, z) & =\alpha^{-4 z} B_{4} B_{2} B_{1} B_{1} \\
& =\alpha^{-4 z} B(4 z, 1-4 z) B(2 z, 1-2 z) B^{2}(z, 1-z) .
\end{aligned}
$$

These types of models exemplify a rich structure capturing some aspects of real QFT calculations, and we refer the reader to $[4,32,37,34]$ for more details on such toy models.

For latter use, we parameterize 't Hooft's mass

$$
\mu \longrightarrow \mu(s):=\mathrm{e}^{s} \mu, s \in \mathbb{R},
$$

such that $\alpha:=a / \mu \rightarrow \alpha(s)$, and for a fixed, $a$, we define

$$
\varphi(a, \mu, z) \rightarrow \varphi(\alpha(s), z)=: \varphi(s, z) .
$$

\subsection{Connes-Kreimer's Birkhoff decomposition of Hopf algebra characters}

Connes and Kreimer extended the work by Kreimer [31,32] and established the connected graded commutative non-cocommutative Hopf algebra of Feynman graphs corresponding to a perturbative quantum field theory (pQFT).

Moreover, in the context of minimal subtraction as renormalization scheme in dimensional regularization, Connes and Kreimer [9,10] discovered a unique Birkhoff type decomposition of Hopf algebra characters with values in the $\mathbb{C}$-algebra $\mathcal{A}$ of meromorphic functions capturing the process 
of renormalization in pQFT. Namely, for any $\varphi \in G_{\mathcal{A}}$, we have

$$
\varphi=\varphi_{-}^{\star-1} \star \varphi_{+},
$$

where both $\varphi_{-}$and $\varphi_{+}$belong to $G_{\mathcal{A}}$, and $\varphi_{+}(x) \in \mathcal{A}_{+}$for any $x \in \mathcal{H}$, whereas $\varphi_{-}(x) \in \mathcal{A}_{-}$for any $x \in \operatorname{Ker} \epsilon$. Here, $\mathcal{A}_{-}$is the algebra of polynomials in $z^{-1}$ without constant term (the "pole parts"), and $\mathcal{A}_{+}$is the algebra of meromorphic functions which are holomorphic at $z=0$, corresponding to the splitting of the $\mathbb{C}$-algebra $\mathcal{A}=\mathcal{A}_{+} \oplus \mathcal{A}_{-}$of meromorphic functions. We denote by $\pi: \mathcal{A} \rightarrow \mathcal{A}_{-}$the projection onto $\mathcal{A}_{-}$.

The components $\varphi_{-}$and $\varphi_{+}$are given by recursive formulas. Suppose that $\varphi_{-}(x)$ and $\varphi_{+}(x)$ are known for $x \in \mathcal{H}^{(n-1)}$. Define for $x \in \mathcal{H}^{(n)}$ Bogoliubov's preparation map:

$$
\bar{R}: x \longmapsto \varphi_{-} \star(\varphi-e)(x)=\varphi(x)+\sum_{(x)} \varphi_{-}\left(x^{\prime}\right) \varphi\left(x^{\prime \prime}\right) .
$$

The components in the Birkhoff decomposition are then given by

$$
\begin{aligned}
& \varphi_{-}(x)=-\pi(\bar{R}(x)), \\
& \varphi_{+}(x)=\left(I d_{\mathcal{A}}-\pi\right)(\bar{R}(x)) .
\end{aligned}
$$

The factor $\varphi_{+}(x)$ is the renormalized character, whereas $\varphi_{-}(x)$ is the sum of counter terms one must add to $\bar{R}(x)$ to get $\varphi_{+}(x)$. In the example of minimal subtraction scheme, the renormalized value of the character $\varphi$ at $0 \in \mathbb{C}$ is the well-defined complex number $\varphi_{+}(0)$, whereas $\varphi(0)$ may not exist. The fact that $\varphi_{-}$and $\varphi_{+}$are still characters relies on the Rota-Baxter property for the projection $\pi$, see e.g., $[17,18,21]$. Moreover, Connes-Kreimer's results do not depend on the type of regularization or subtraction scheme.

In terms of the toy-model character $\varphi(s, z)=\varphi\left(a, \mathrm{e}^{s} \mu, z\right)$, see equation (2.13), with parameterized 't Hooft mass (2.14), mapping the Hopf algebra $\mathcal{H}_{\mathcal{T}}$ of non-planar integer decorated rooted trees to $\left.\mathcal{A}:=\mathbb{C}\left[z^{-1}, z\right]\right]$ $[[\log (\alpha(s))]], \alpha(s):=a /\left(\mathrm{e}^{s} \mu\right)>0$, which decomposes into

$$
\mathcal{A}=z^{-1} \mathbb{C}\left[z^{-1}\right][[\log (\alpha(s))]] \oplus \mathbb{C}[[z]][[\log (\alpha(s))]],
$$

we find for the primitive tree $\bullet \in \mathcal{H}^{(1)}$ the counter term

$$
\begin{aligned}
\varphi_{-}(\bullet)(s, z) & =-\pi(\bar{R}(\bullet))=-\pi(\varphi(\bullet)) \\
& =-\pi\left(\alpha(s)^{-z} B_{1}\right) \in z^{-1} \mathbb{C}\left[z^{-1}\right],
\end{aligned}
$$

and for $\therefore \in \mathcal{H}^{(3)}$, we find

$$
\begin{aligned}
\bar{R}\left({ }^{\prime}\right) & =\varphi\left({ }^{\prime}\right)+2 \varphi_{-}(\bullet) \varphi(\bullet)+\varphi_{-}(\bullet) \varphi_{-}(\bullet) \\
& =\alpha^{-3 z} B_{3} B_{1}^{2}-2 \pi\left(\alpha^{-z} B_{1}\right) \alpha^{-2 z} B_{2} B_{1}+\pi\left(\alpha^{-2 z} B_{1}^{2}\right) \alpha^{-z} B_{1},
\end{aligned}
$$


such that the counter term is given by

$$
\varphi_{-}(\diamond)(s, z)=-\pi\left(\varphi(\diamond)+2 \varphi_{-}(\bullet) \varphi(\bullet)+\varphi_{-}(\bullet) \varphi_{-}(\bullet)\right) .
$$

A detailed calculation shows that $\varphi_{-}\left(\curvearrowright_{\circ}\right)(s, z) \in z^{-1} \mathbb{C}\left[z^{-1}\right]$. Hence any dependence on $\mu$ has disappeared. Feynman rule characters in dimensional regularization depend on the unit of mass $\mu$. However, for the corresponding counter terms in their Birkhoff decomposition, it is true in general that $\varphi_{-}(t)(s, z) \in z^{-1} \mathbb{C}\left[z^{-1}\right], t \in \mathcal{H}_{\mathcal{T}}$. In terms of our toy-model character $(2.13)$ with parameterized unit mass $\mu=\mu(s)$ (2.14), this may be summarized by saying that $\partial_{s} \varphi_{-}(t)(s, z)=0, t \in \mathcal{H}_{\mathcal{T}}$. We will come back to this in Section 3 .

The reader should compare the above counter term with the expression for $S\left(\Omega_{0}\right)$ in equation (2.11). Eventually, the corresponding renormalized expression $\varphi_{+}\left(\AA_{\bullet}\right):=\left(I d_{\mathcal{A}}-\pi\right)\left(\bar{R}\left(\AA_{\diamond}\right)\right)$ is then given by

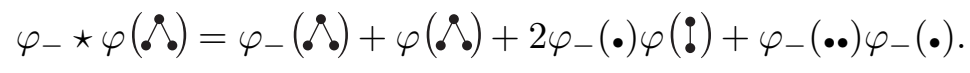

The reader should verify that $\varphi_{-}(\bullet \bullet)=\varphi_{-}(\bullet) \varphi_{-}(\bullet)$, that is, $\varphi_{-} \in G_{\mathcal{A}}$, hence $\varphi_{+}=\varphi_{-} \star \varphi \in G_{\mathcal{A}}$.

\subsection{The matrix representation}

In this section, we recall the matrix representation of $\mathcal{L}(\mathcal{H}, \mathcal{A})$ associated with a left coideal. The next step consists in understanding the beta-function of Connes-Kreimer [11] in this matrix setting. We follow Sakakibara's approach [43], giving his clever computations the concrete support of triangular matrices.

Let us start by retrieving some material from [20,21,23]. Recall that the subalgebra $\mathcal{M}_{n}^{\ell}(\mathcal{A}) \subset \mathcal{M}_{n}(\mathcal{A})$ of lower triangular matrices in the algebra of $n \times n$ matrices with entries in the algebra $\mathcal{A}$ and with $n$ finite or infinite has a decreasing filtration and is complete in the induced topology. Indeed, $\mathcal{M}_{n}^{\ell}(\mathcal{A})^{m}$ is the ideal of strictly lower triangular matrices with zero on the main diagonal and on the first $m-1$ subdiagonals, $m>1$. We then have the decreasing filtration

$$
\mathcal{M}_{n}^{\ell}(\mathcal{A}) \supset \mathcal{M}_{n}^{\ell}(\mathcal{A})^{1} \supset \cdots \supset \mathcal{M}_{n}^{\ell}(\mathcal{A})^{m-1} \supset \mathcal{M}_{n}^{\ell}(\mathcal{A})^{m} \supset \cdots, m<n,
$$

with

$$
\mathcal{M}_{n}^{\ell}(\mathcal{A})^{u} \mathcal{M}_{n}^{\ell}(\mathcal{A})^{v} \subset \mathcal{M}_{n}^{\ell}(\mathcal{A})^{u+v}
$$


For $\mathcal{A}$ being commutative, we denote by $\mathfrak{M}_{n}(\mathcal{A})$ the group of lower triangular matrices with unit diagonal which is $\mathfrak{M}_{n}(\mathcal{A})=\mathbf{1}+\mathcal{M}_{n}^{\ell}(\mathcal{A})^{1}$. Here the $n \times n$, $n \leq \infty$, unit matrix is given by

$$
\mathbf{1}:=\left(\delta_{i j} 1_{\mathcal{A}}\right)_{1 \leq i, j \leq n} .
$$

Let $\mathcal{H}$ be a connected filtered Hopf algebra over $k$, let $\mathcal{A}$ be any commutative unital $k$-algebra and let $(\mathcal{L}(\mathcal{H}, \mathcal{A}), \star)$ be the algebra of $k$-linear maps from $\mathcal{H}$ to $\mathcal{A}$ endowed with the convolution product. Let $J$ be any left coideal of $\mathcal{H}$ (i.e., a vector subspace of $\mathcal{H}$ such that $\Delta(J) \subset \mathcal{H} \otimes J$ ).

We fix a basis $X=\left(x_{i}\right)_{i \in I}$ of the left coideal $J$ (a left subcoset in the terminology of [20]). We suppose further that this basis is denumerable (hence indexed by $I=\mathbb{N}$ or $I=\{1, \cdots, m\}$ ) and filtration ordered, i.e., such that if $i \leq j$ and $x_{j} \in \mathcal{H}^{(n)}$, then $x_{i} \in \mathcal{H}^{(n)}$.

Definition 2.2. The coproduct matrix in the basis $X$ is the $|I| \times|I|$ matrix $M$ with entries in $\mathcal{H}$ defined by

$$
\Delta\left(x_{i}\right)=\sum_{j \in I} M_{i j} \otimes x_{j} .
$$

Lemma 2.3. The coproduct matrix is lower triangular with diagonal terms equal to 1 .

Proof. Suppose $x_{i} \in \mathcal{H}^{(n)}$ and $x_{i} \notin \mathcal{H}^{(n-1)}$. Then it is well known that (see e.g., $[25],[39])$ :

$$
\Delta\left(x_{i}\right)=x_{i} \otimes 1+1 \otimes x_{i}+\text { terms of filtration degree } \leq n-1 .
$$

Then clearly $M_{i i}=1$, and moreover if $M_{i j} \neq 0$ and $i \neq j$, then $x_{j} \in \mathcal{H}^{(n-1)}$. If $i<j$, this implies $x_{i} \in H^{(n-1)}$, which contradicts the hypothesis. Hence $M_{i j}=0$ if $i<j$.

Recalling the example of rooted trees and choosing the following subset $\mathcal{T}^{\prime} \subset \mathcal{T}$ of rooted trees with the displayed linear order

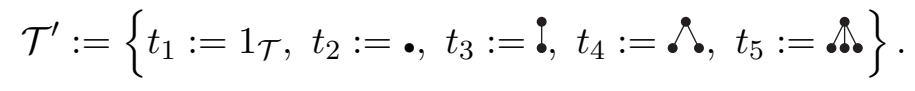

The $5 \times 5$ coproduct matrix of Definition 2.2 is then given by

$$
M=\left(\begin{array}{ccccc}
1_{\mathcal{T}} & 0 & 0 & 0 & 0 \\
\bullet & 1_{\mathcal{T}} & 0 & 0 & 0 \\
\vdots & \bullet & 1_{\mathcal{T}} & 0 & 0 \\
\AA & \bullet & 2 \bullet & 1_{\mathcal{T}} & 0 \\
\AA & \cdots & 3 \bullet & 3 \bullet & 1_{\mathcal{T}}
\end{array}\right)
$$


Observe that for each tree $t_{i} \in \mathcal{T}^{\prime}$, all cotrees in $\bar{\Delta}\left(t_{i}\right)$ are of degree strictly lower than $\operatorname{deg}\left(t_{i}\right)$ and are contained in $\mathcal{T}^{\prime}$, hence $\mathcal{T}^{\prime}$ forms a left coideal in $\mathcal{H}_{\mathcal{T}}$.

Now define $\Psi_{J}: \mathcal{L}(\mathcal{H}, \mathcal{A}) \rightarrow \operatorname{End}_{\mathcal{A}}(\mathcal{A} \otimes J)$ by

$$
\Psi_{J}[f]\left(x_{j}\right)=\sum_{i} f\left(M_{i j}\right) \otimes x_{i}
$$

In other words, the matrix of $\Psi_{J}[f]$ is given by $\left(f\left(M_{i j}\right)\right)_{i, j \in I}$.

Proposition 2.4. The map $\Psi_{J}$ defined above is an algebra homomorphism. Its transpose does not depend on the choice of the basis.

Proof. The second statement is straightforward: let us denote by ${ }^{\top} \Psi_{J}$ the transpose of $\Psi_{J}$, i.e., the map defined by

$$
{ }^{\top} \Psi_{J}[f]\left(x_{i}\right)=\sum_{j} f\left(M_{i j}\right) \otimes x_{j} .
$$

For any $x \in J$ we have then, using Sweedler's notation,

$$
{ }^{\top} \Psi_{J}[f](x)=\sum_{(x)} f\left(x_{(1)}\right) \otimes x_{(2)} .
$$

Hence, ${ }^{\top} \Psi_{J}[f]$ has an intrinsic expression as the composition of the three maps below:

$$
\mathcal{A} \otimes J \stackrel{I d_{\mathcal{A}} \otimes \Delta}{\longrightarrow} \mathcal{A} \otimes \mathcal{H} \otimes J \stackrel{I d_{\mathcal{A}} \otimes f \otimes I d_{J}}{\longrightarrow} \mathcal{A} \otimes \mathcal{A} \otimes J \stackrel{m_{\mathcal{A}} \otimes I d_{J}}{\longrightarrow} \mathcal{A} \otimes J .
$$

We have to show for any $f, g \in \mathcal{L}(\mathcal{H}, \mathcal{A})$,

$$
\Psi_{J}[f \star g]=\Psi_{J}[f] \Psi_{J}[g] .
$$

It is shown in [20] that ${ }^{\top} \Psi_{J}$ is an anti-homomorphism, which proves the claim. We give here an alternative proof. Using coassociativity $(\operatorname{Id} \otimes \Delta) \circ$ $\Delta\left(x_{i}\right)=(\Delta \otimes I d) \circ \Delta\left(x_{i}\right)$, we immediately get

$$
\Delta\left(M_{i j}\right)=\sum_{k=1}^{|I|} M_{i k} \otimes M_{k j} .
$$

Hence,

$$
\begin{aligned}
\Psi_{J}[f \star g]\left(x_{j}\right) & =\sum_{i}(f \star g)\left(M_{i j}\right) \otimes x_{i} \\
& =\sum_{i} \sum_{k} f\left(M_{i k}\right) g\left(M_{k j}\right) \otimes x_{i} \\
& =\Psi_{J}[f] \Psi_{J}[g]\left(x_{j}\right),
\end{aligned}
$$

which proves (2.23). 
The Lie algebra of infinitesimal characters is mapped into a Lie subalgebra $\widehat{\mathfrak{g}}_{\mathcal{A}}$ in $\mathcal{M}_{|I|}^{\ell}(\mathcal{A})^{1}$. This is immediately seen by applying definition (2.22), since elements in $\mathfrak{g}_{A}$ map the unit to zero due to relation (2.3). Whereas characters in the group $G_{\mathcal{A}}$ are mapped to the subgroup $\widehat{G}_{\mathcal{A}} \subset \mathfrak{M}_{|I|}(\mathcal{A})$.

The toy-model character $\varphi=\varphi(s, z)$ in equation (2.13) with parameterized 't Hooft mass, applied to the coproduct matrix (2.21) gives

$$
\widehat{\varphi}(s, z)=\left(\begin{array}{ccccc}
1_{\mathcal{A}} & 0 & 0 & 0 & 0 \\
\alpha(s)^{-z} B_{1} & 1_{\mathcal{A}} & 0 & 0 & 0 \\
\alpha(s)^{-2 z} B_{2} B_{1} & \alpha(s)^{-z} B_{1} & 1_{\mathcal{A}} & 0 & 0 \\
\alpha(s)^{-3 z} B_{3} B_{1}^{1} & \alpha(s)^{-2 z} B_{1}^{2} & 2 \alpha(s)^{-z} B_{1} & 1_{\mathcal{A}} & 0 \\
\alpha(s)^{-4 z} B_{4} B_{1}^{3} & \alpha(s)^{-3 z} B_{1}^{3} & 3 \alpha(s)^{-2 z} B_{1}^{2} & 3 \alpha(s)^{-z} B_{1} & 1_{\mathcal{A}}
\end{array}\right) .
$$

Recall that $\alpha=\alpha(s)=a / \mu(s)$, where $\mu(s)=\mathrm{e}^{s} \mu$.

The following remarks should be useful latter. The coproduct matrix $M$ with entries in $\mathcal{H}$ can be seen as the image of the identity map under $\Psi_{J}: \mathcal{L}(\mathcal{H}, \mathcal{H}) \rightarrow \operatorname{End}_{\mathcal{H}}(\mathcal{H} \otimes J)$, i.e.,

$$
\Psi_{J}[I d]\left(x_{j}\right)=\sum_{i} I d\left(M_{i j}\right) \otimes x_{i} .
$$

Equations (2.6) imply for the matrix representation of the antipode $S \in$ $\mathcal{L}(\mathcal{H}, \mathcal{H})$

$$
\begin{aligned}
\Psi_{J}[S \star I d]\left(x_{j}\right) & =\Psi_{J}[S] \circ \Psi_{J}[I d]\left(x_{j}\right) \\
& =\sum_{i} \sum_{k} S\left(M_{i k}\right) \operatorname{Id}\left(M_{k j}\right) \otimes x_{i} \\
& =\Psi_{J}[\eta \circ \epsilon]\left(x_{j}\right) \\
& =\sum_{i} \eta \circ \epsilon\left(M_{i j}\right) \otimes x_{i} \\
& =\sum_{i} \mathbf{1} \delta_{i j} \otimes x_{i} .
\end{aligned}
$$

Here 1 denotes the $|I| \times|I|$ unit matrix, $\left(\delta_{i j} 1_{\mathcal{T}}\right)_{1 \leq i, j \leq|I|}$. Hence, $\Psi_{J}[S]=$ $M^{-1}$, and the inverse can be calculated readily in terms of the geometric series

$$
\Psi_{J}[S]=M^{-1}=\mathbf{1}+\sum_{k>0}(-1)^{k}(M-\mathbf{1})^{k} .
$$

Using the bijection $\exp ^{\star}$ between $\mathfrak{g}_{\mathcal{A}}$ and $G_{\mathcal{A}}$, we may write any $\varphi \in G_{\mathcal{A}}$ as an exponential of the element $\alpha=\log ^{\star}(\varphi)$ in $\mathfrak{g}_{\mathcal{A}}$. In terms of matrices, we 
find $\log (\Psi[\varphi])=\varphi(\log (\Psi[I d]))$, using the fact that $\varphi$ is a character, and

$$
\log (\Psi[I d])=\log (M)=\sum_{k>0}(-1)^{k} \frac{(M-\mathbf{1})^{k}}{k}
$$

defines the matrix of the so-called normal coordinates.

\subsection{The matrix form of Connes-Kreimer's Birkhoff decomposition}

Suppose that the commutative target space algebra $\mathcal{A}$ in $\mathcal{L}(\mathcal{H}, \mathcal{A})$ splits into two subalgebras

$$
\mathcal{A}=\mathcal{A}_{-} \oplus \mathcal{A}_{+},
$$

where the unit $1_{\mathcal{A}}$ belongs to $\mathcal{A}_{+}$. Let us denote by $\pi: \mathcal{A} \rightarrow \mathcal{A}_{-}$the projection onto $\mathcal{A}_{-}$parallel to $\mathcal{A}_{+}$. One readily verifies that $\pi$ is an idempotent Rota-Baxter operator [21], that is, it satisfies the relation

$$
\pi(\pi(a) b+a \pi(b)-a b)=\pi(a) \pi(b) .
$$

Indeed, let $a, b \in \mathcal{A}$

$$
\pi(a) b+a \pi(b)-a b=\pi(a) \pi(b)-\left(I d_{\mathcal{A}}-\pi\right)(a)\left(I d_{\mathcal{A}}-\pi\right)(b),
$$

such that applying $\pi$ on both sides gives relation (2.26), since it eliminates the term $\left(I d_{\mathcal{A}}-\pi\right)(a)\left(I d_{\mathcal{A}}-\pi\right)(b)$ without changing the term $\pi(a) \pi(b)$, as $\pi\left(I d_{\mathcal{A}}-\pi\right)(a)=0$ and $\mathcal{A}_{-}=\pi(\mathcal{A}), \mathcal{A}_{+}=\left(I d_{\mathcal{A}}-\pi\right)(\mathcal{A})$ are subalgebras.

In fact, this is a special case of an additive decomposition theorem characterizing Rota-Baxter algebras, which was proven by Atkinson for general, not necessarily associative algebras in [2].

Theorem 2.5 [2]. Let $\mathcal{A}$ be a k-algebra. A k-linear operator $R: \mathcal{A} \rightarrow \mathcal{A}$ satisfies the Rota-Baxter relation (2.26) if and only if the following two statements are true. Firstly, $\mathcal{A}_{-}:=R(\mathcal{A})$ and $\mathcal{A}_{+}:=\tilde{R}(\mathcal{A})$ are subalgebras in $\mathcal{A}$. Secondly, for $x, y, z \in \mathcal{A}, R(x) R(y)=R(z)$ implies $\tilde{R}(x) \tilde{R}(y)=-\tilde{R}(z)$. Here we denoted the map $\tilde{R}:=\left(I d_{\mathcal{A}}-R\right)$.

The case of an idempotent Rota-Baxter map implies $\mathcal{A}_{-} \cap \mathcal{A}_{+}=\{0\}$. In the context of perturbative renormalization in QFT where the regularization prescription implies the target space algebra $\mathcal{A}$, the corresponding splitting of $\mathcal{A}$ into a direct sum of two subalgebras is called a renormalization scheme. For example, the minimal renormalization scheme in dimensional regularization corresponds to the splitting of the $\mathbb{C}$-algebra $\mathcal{A}$ of meromorphic functions in which $\mathcal{A}_{-}$is the algebra of polynomials in $z^{-1}$ without constant term (the "pole parts"), and $\mathcal{A}_{+}$is the algebra of meromorphic functions which are holomorphic at $z=0$. 
The following theorem describes a multiplicative decomposition for associative unital Rota-Baxter algebras and was observed by Atkinson in [2], see also [23].

Theorem $2.6[2]$. Let $\mathcal{A}$ be an associative unital Rota-Baxter algebra with Rota-Baxter map $R$. Suppose $\mathcal{A}$ to have a decreasing filtration and to be complete in the induced topology. Assume $X$ and $Y$ in $\mathcal{A}$ to be solutions of the equations

$$
X=1_{\mathcal{A}}-R(X a) \quad \text { and } \quad Y=1_{\mathcal{A}}-\tilde{R}(a Y),
$$

for $a \in \mathcal{A}^{(1)}$. Then we have the following factorization

$$
X\left(1_{\mathcal{A}}+a\right) Y=1_{\mathcal{A}}, \quad \text { such that } 1_{\mathcal{A}}+a=X^{-1} Y^{-1} .
$$

For an idempotent Rota-Baxter map, this factorization is unique.

Proof. The Rota-Baxter relation (2.26) yields for any $\alpha, \beta \in \mathcal{A}$

$$
R(\alpha \tilde{R}(\beta))+\tilde{R}(R(\alpha) \beta)=R(\alpha) \tilde{R}(\beta) .
$$

We then simply calculate the product $X Y$ and use equation (2.29), with $\alpha=X a$ and $\beta=a Y$ :

$$
\begin{aligned}
X Y & =\left(1_{\mathcal{A}}-R(X a)\right)\left(1_{\mathcal{A}}-\tilde{R}(a Y)\right) \\
& =1_{\mathcal{A}}-R(X a)-\tilde{R}(a Y)+R(X a) \tilde{R}(a Y) \\
& =1_{\mathcal{A}}-R\left(X a\left(1_{\mathcal{A}}-\tilde{R}(a Y)\right)\right)-\tilde{R}\left(\left(1_{\mathcal{A}}-R(X a)\right) a Y\right) \\
& =1_{\mathcal{A}}-R(X a Y)-\tilde{R}(X a Y) \\
& =1_{\mathcal{A}}-X a Y .
\end{aligned}
$$

Hence, we obtain the factorization in (2.28). The uniqueness for an idempotent Rota-Baxter map is easy to show $[18,23]$.

Let us come back to the matrix representation of $\mathcal{L}(\mathcal{H}, \mathcal{A})$ with a splitting $\mathcal{A}(2.25)$ via $\Psi_{J}(2.22)$. We define a Rota-Baxter map $\mathrm{R}$ on $\Psi_{J}[\mathcal{L}] \subset$ $\mathcal{M}_{|I|}^{\ell}(\mathcal{A})$ by extending the Rota-Baxter map $\pi$ on $\mathcal{A}$ entrywise, i.e., for the matrix $\tau=\left(\tau_{i j}\right) \in \mathcal{M}_{|I|}^{\ell}(\mathcal{A})$, define

$$
\mathrm{R}(\tau)=\left(\pi\left(\tau_{i j}\right)\right) .
$$

Theorem $2.7[20]$. Then the triple $\left(\mathcal{M}_{|I|}^{\ell}(\mathcal{A}), \mathrm{R},\left\{\mathcal{M}_{|I|}^{\ell}(\mathcal{A})^{l}\right\}_{l<|I|}\right)$ forms a non-commutative complete filtered Rota-Baxter algebra with idempotent Rota-Baxter map R. 
Atkinson's multiplicative decomposition immediately implies a factorization of $\widehat{G}_{\mathcal{A}} \subset \mathfrak{M}_{|I|}^{\ell}(\mathcal{A})$ into the subgroups:

$$
\widehat{G}_{\mathcal{A}}^{-} \subset \mathbf{1}+\mathrm{R}\left(\mathcal{M}_{|I|}^{\ell}(\mathcal{A})^{1}\right) \subset \mathfrak{M}_{|I|}^{\ell}(\mathcal{A})
$$

and

$$
\widehat{G}_{\mathcal{A}}^{+} \subset \mathbf{1}+\tilde{\mathrm{R}}\left(\mathcal{M}_{|I|}^{\ell}(\mathcal{A})^{1}\right) \subset \mathfrak{M}_{|I|}^{\ell}(\mathcal{A}),
$$

that is, for each $\widehat{\varphi}:=\Psi[\varphi] \in \widehat{G}_{\mathcal{A}}, \varphi \in G_{\mathcal{A}}$, there exist unique $\widehat{\varphi}_{-} \in \widehat{G}_{\mathcal{A}}^{-}$and $\widehat{\varphi}_{+} \in \widehat{G}_{\mathcal{A}}^{+}$such that

$$
\widehat{\varphi}=\widehat{\varphi}_{-}^{-1} \widehat{\varphi}_{+}
$$

We immediately see that $\widehat{\varphi}_{-}$and $\widehat{\varphi}_{+}^{-1}$ are unique solutions of the equations (2.27) in Theorem 2.6:

$$
\begin{aligned}
& \widehat{\varphi}_{-}=\mathbf{1}-\mathrm{R}\left(\widehat{\varphi}_{-}(\widehat{\varphi}-\mathbf{1})\right), \\
& \widehat{\varphi}_{+}^{-1}=\mathbf{1}-\tilde{\mathrm{R}}\left((\widehat{\varphi}-\mathbf{1}) \widehat{\varphi}_{+}^{-1}\right) .
\end{aligned}
$$

Moreover, after some simple algebra using the factorization $\widehat{\varphi}=\widehat{\varphi}_{-}^{-1} \widehat{\varphi}_{+}$

$$
\widehat{\varphi}_{+}\left(\widehat{\varphi}^{-1}-\mathbf{1}\right)=\widehat{\varphi}_{-}-\widehat{\varphi}_{+}=-\widehat{\varphi}_{-}(\widehat{\varphi}-\mathbf{1}),
$$

we immediately get the recursion for $\widehat{\varphi}_{+}[20]$ :

$$
\widehat{\varphi}_{+}=\mathbf{1}-\tilde{\mathrm{R}}\left(\widehat{\varphi}_{+}\left(\widehat{\varphi}^{-1}-\mathbf{1}\right)\right) .
$$

and hence we see that

$$
\widehat{\varphi}_{+}=\mathbf{1}+\tilde{\mathrm{R}}\left(\widehat{\varphi}_{-}(\widehat{\varphi}-\mathbf{1})\right)
$$

The matrix entries of $\widehat{\varphi}_{-}$and $\widehat{\varphi}_{+}^{-1}$ can be calculated without recursions using $\sigma:=\widehat{\varphi}$ from the equations:

$$
\begin{aligned}
& \left(\widehat{\varphi}_{-}\right)_{i j}=-\pi\left(\sigma_{i j}\right) \\
& -\sum_{k=2}^{j-i} \sum_{i>l_{1}>l_{2}>\cdots>l_{k-1}>j}(-1)^{k+1} \pi\left(\pi\left(\cdots \pi\left(\sigma_{i l_{1}}\right) \sigma_{l_{1} l_{2}}\right) \cdots \sigma_{l_{k-1} j}\right) \\
& \left(\widehat{\varphi}_{+}^{-1}\right)_{i j}=-\tilde{\pi}\left(\left(\sigma^{-1}\right)_{i j}\right) \\
& -\sum_{k=2}^{j-i} \sum_{i>l_{1}>l_{2}>\cdots>l_{k-1}>j}(-1)^{k+1} \tilde{\pi}\left(\tilde{\pi}\left(\cdots \tilde{\pi}\left(\left(\sigma^{-1}\right)_{i l_{1}}\right)\left(\sigma^{-1}\right)_{l_{1} l_{2}}\right) \cdots\left(\sigma^{-1}\right)_{l_{k-1} j}\right),
\end{aligned}
$$

where $\tilde{\pi}:=I d_{\mathcal{A}}-\pi$. The matrix entries of $\widehat{\varphi}_{+}$follow from the first formula, i.e., the one for the entries in $\widehat{\varphi}_{-}$, by replacing $\pi$ by $-\tilde{\pi}$. We may therefore 
define the matrix:

$$
\widehat{\bar{R}}[\varphi]:=\widehat{\varphi}-(\widehat{\varphi}-\mathbf{1})
$$

such that

$$
\widehat{\varphi}_{-}=\mathbf{1}-\mathrm{R}(\widehat{\bar{R}}[\varphi]) \quad \text { and } \quad \widehat{\varphi}_{+}=\mathbf{1}+\tilde{\mathrm{R}}(\widehat{\widehat{R}}[\varphi]) .
$$

In fact, equations (2.35) and (2.36) may be called Bogoliubov's matrix formulae for the counter term and renormalized Feynman rules matrix, $\widehat{\varphi}_{-}$, $\widehat{\varphi}_{+}$, respectively. Equation (2.36) is the matrix form of Bogoliubov's $\bar{R}$ - or preparation map (2.16), e.g., see [13]:

$$
\widehat{\bar{R}}[\varphi]:=\Psi_{J}[\bar{R}]=\Psi_{J}\left[\varphi_{-} \star(\varphi-e)\right] .
$$

Here, $\varphi_{-}$is the counter term character (2.17) of the algebraic Birkhoff decomposition of Connes and Kreimer $[9,10]$, which we mentioned in the foregoing section. To phrase it differently, the above matrix factorization follows via the representation $\Psi_{J}$ from Connes-Kreimer's Birkhoff factorization (2.15) on the group $G_{\mathcal{A}}$ of $\mathcal{A}$-valued Hopf algebra characters.

Remark 2.8. We may apply the result from [17, 18, 20, 23], see also [39], to the above matrix representation of $\mathfrak{g}_{\mathcal{A}}$ respectively $G_{\mathcal{A}}$. In these references, a unique non-linear map $\chi$ was established on $\mathfrak{g}_{\mathcal{A}}$, which allows to write the characters $\varphi_{-}$and $\varphi_{+}$as exponentials. In the matrix picture, we hence find for $\widehat{Z} \in \widehat{\mathfrak{g}}_{\mathcal{A}}$ and $\widehat{\varphi}=\exp (\widehat{Z}) \in \widehat{G}_{\mathcal{A}}$,

$$
\widehat{\varphi}=\exp (\mathrm{R}(\chi(\widehat{Z}))) \exp (\tilde{\mathrm{R}}(\chi(\widehat{Z}))) .
$$

The matrices $\widehat{\varphi}_{-}:=\exp (-\mathrm{R}(\chi(\widehat{Z})))$ and $\widehat{\varphi}_{+}^{-1}:=\exp (-\tilde{\mathrm{R}}(\chi(\widehat{Z})))$ are in $\widehat{G}_{\mathcal{A}}^{-}$ and $\widehat{G}_{\mathcal{A}}^{+}$, respectively, and solve Bogoliubov's matrix formulae in (2.37).

\section{The matrix representation of the beta-function}

It is the goal of this section to establish a matrix representation of the betafunction as it appears in the work of Connes and Kreimer [11]. Once we have achieved this, we reformulate in a transparent manner Sakakibara's findings [43] hereby providing a firm ground for his calculations. In the next paragraph, we review the main points of the beta-function calculus in the Hopf algebra context following mainly the paper [39].

\subsection{The beta-function in the Hopf algebra of renormalization}

From now on, $k=\mathbb{C}$ stands for the complex numbers, and $\mathcal{A}$ will denote the algebra of meromorphic functions in one complex variable $z$ endowed with 
the minimal subtraction scheme. Hence, $\mathcal{A}=\mathcal{A}_{-} \oplus \mathcal{A}_{+}$, where $\mathcal{A}_{+}$is the subalgebra of meromorphic functions which are holomorphic at $z=0$, and $\mathcal{A}_{-}$stands for the polynomials in $z^{-1}$ without constant term. We moreover suppose that the Hopf algebra $\mathcal{H}$ is graded, with filtration coming from the graduation, i.e.,

$$
\mathcal{H}^{(n)}=\bigoplus_{0 \leq k \leq n} \mathcal{H}_{k}
$$

The grading induces a biderivation $Y$ defined on homogeneous elements by

$$
\begin{aligned}
Y & : \mathcal{H}_{n} \longrightarrow \mathcal{H}_{n} \\
& x \longmapsto n x .
\end{aligned}
$$

Exponentiating $Y$, we get a one-parameter group $\theta_{t}$ of automorphisms of the Hopf algebra $\mathcal{H}$, defined on $\mathcal{H}_{n}$ by

$$
\theta_{t}(x)=\mathrm{e}^{n t} x .
$$

The map $\varphi \mapsto \varphi \circ Y$ is a derivation of $(\mathcal{L}(\mathcal{H}, \mathcal{A}), \star)$, and $\varphi \mapsto \varphi \circ \theta_{t}$ is an automorphism of $(\mathcal{L}(\mathcal{H}, \mathcal{A}), \star)$ for any complex $t$. We will rather consider the one-parameter group $\varphi \mapsto \varphi \circ \theta_{t z}$ of automorphisms of the algebra $(\mathcal{L}(\mathcal{H}, \mathcal{A}), \star)$, i.e.,

$$
\varphi^{t}(x)(z):=\mathrm{e}^{t z|x|} \varphi(x)(z) .
$$

Recall that $|x|:=\operatorname{deg}(x)$, for $x \in H$. Differentiating at $t=0$, we get

$$
\left.\frac{d}{d t}\right|_{t=0} \varphi^{t}=z(\varphi \circ Y)
$$

We denote by $G_{\mathcal{A}}^{\Phi}$ the set of those characters $\varphi \in G$ such that the negative part of the Birkhoff decomposition of $\varphi^{t}$ does not depend on $t$, namely

$$
G_{\mathcal{A}}^{\Phi}=\left\{\varphi \in G_{\mathcal{A}} \mid \frac{d}{d t}\left(\varphi^{t}\right)_{-}=0\right\}
$$

In particular, the dimensional-regularized Feynman rules verify this property: in physical terms, the counter terms do not depend on the choice of the arbitrary mass-parameter $\mu$ ('t Hooft's mass) one must introduce in dimensional regularization in order to get dimensionless expressions (see [10]). We also denote by $G_{\mathcal{A}_{-}}^{\Phi}$ the elements $\varphi$ of $G_{\mathcal{A}}^{\Phi}$ such that $\varphi=\varphi_{-}^{\star-1}$. Recall from [39] that there is a bijection $\widetilde{R}: G_{\mathcal{A}} \rightarrow \mathfrak{g}_{\mathcal{A}}$ defined by

$$
\varphi \circ Y=\varphi \star \widetilde{R}(\varphi) .
$$

Since composition on the right with $Y$ is a derivation for the convolution product, the map $\widetilde{R}$ verifies a cocycle property

$$
\widetilde{R}(\varphi \star \psi)=\widetilde{R}(\psi)+\psi^{\star-1} \star \widetilde{R}(\varphi) \star \psi .
$$


We summarize some key results of [11] in the following proposition:

\section{Proposition 3.1.}

(1) For any $\varphi \in G_{\mathcal{A}}$, there is a one-parameter family $h_{t}$ in $G_{\mathcal{A}}$ such that $\varphi^{t}=\varphi \star h_{t}$, and we have

$$
\dot{h}_{t}:=\frac{d}{d t} h_{t}=h_{t} \star z \widetilde{R}\left(h_{t}\right)+z \widetilde{R}(\varphi) \star h_{t} .
$$

(2) $z \widetilde{R}$ restricts to a bijection from $G_{\mathcal{A}}^{\Phi}$ onto $\mathfrak{g}_{\mathcal{A}} \cap \mathcal{L}\left(\mathcal{H}, \mathcal{A}_{+}\right)$. Moreover, it is a bijection from $G_{\mathcal{A}_{-}}^{\Phi}$ onto those elements of $\mathfrak{g}_{\mathcal{A}}$ with values in the constants, i.e.,

$$
\mathfrak{g}_{\mathcal{A}}^{c}=\mathfrak{g}_{\mathcal{A}} \cap \mathcal{L}(\mathcal{H}, \mathbb{C}) .
$$

(3) For $\varphi \in G_{\mathcal{A}}^{\Phi}$, the constant term of $h_{t}$, defined by

$$
F_{t}(x)=\lim _{z \rightarrow 0} h_{t}(x)(z),
$$

is a one-parameter subgroup of $G_{\mathcal{A}} \cap \mathcal{L}(\mathcal{H}, \mathbb{C})$, the scalar-valued characters of $\mathcal{H}$.

Proof. For any $\varphi \in G_{\mathcal{A}}$, one can write:

$$
\varphi^{t}=\varphi \star h_{t}
$$

with $h_{t} \in G_{\mathcal{A}}$. From (3.8), (3.3), and (3.4), we immediately get

$$
\varphi \star \dot{h}_{t}=\varphi \star h_{t} \star z \widetilde{R}\left(\varphi \star h_{t}\right) .
$$

Equation (3.6) then follows from the cocycle property (3.5). This proves the first assertion.

Now take any character $\varphi \in G_{\mathcal{A}}^{\Phi}$ with Birkhoff decomposition $\varphi=\varphi_{-}^{\star-1} \star$ $\varphi_{+}$and write the Birkhoff decomposition of $\varphi^{t}$ :

$$
\begin{aligned}
\varphi^{t} & =\left(\varphi^{t}\right)_{-}^{\star-1} \star\left(\varphi^{t}\right)_{+} \\
& =\left(\varphi_{-}\right)^{\star-1} \star\left(\varphi^{t}\right)_{+} \\
& =\left(\varphi \star \varphi_{+}^{\star-1}\right) \star\left(\varphi^{t}\right)_{+} \\
& =\varphi \star h_{t},
\end{aligned}
$$

with $h_{t}$ taking values in $\mathcal{A}_{+}$. Then $z \widetilde{R}(\varphi)$ also takes values in $\mathcal{A}_{+}$, as a consequence of equation (3.6) at $t=0$. Conversely, suppose that $z \widetilde{R}(\varphi)$ takes values in $\mathcal{A}_{+}$. We show that $h_{t}$ also takes values in $\mathcal{A}_{+}$for any $t$, which immediately implies that $\varphi$ belongs to $G_{\mathcal{A}}^{\Phi}$. 
For any $\gamma \in \mathfrak{g}_{\mathcal{A}}$, let us introduce the linear transformation $U_{\gamma}$ of $\mathfrak{g}_{\mathcal{A}}$ defined by

$$
U_{\gamma}(\delta):=\gamma \star \delta+z \delta \circ Y
$$

If $\gamma$ belongs to $\mathfrak{g}_{\mathcal{A}} \cap \mathcal{L}\left(\mathcal{H}, \mathcal{A}_{+}\right)$, then $U_{\gamma}$ restricts to a linear transformation of $\mathfrak{g}_{\mathcal{A}} \cap \mathcal{L}\left(\mathcal{H}, \mathcal{A}_{+}\right)$.

Lemma 3.2. For any $\varphi \in G_{\mathcal{A}}, n \in \mathbb{N}$, we have,

$$
z^{n} \varphi \circ Y^{n}=\varphi \star U_{z \widetilde{R}(\varphi)}^{n}(\mathrm{e}) .
$$

Proof. Case $n=0$ is obvious, $n=1$ is just the definition of $\widetilde{R}$. We check thus by induction, using again the fact that composition on the right with $Y$ is a derivation for the convolution product:

$$
\begin{aligned}
z^{n+1} \varphi \circ Y^{n+1} & =z\left(z^{n} \varphi \circ Y^{n}\right) \circ Y \\
& =z\left(\varphi \star U_{z \widetilde{R}(\varphi)}^{n}(e)\right) \circ Y \\
& =z(\varphi \circ Y) \star U_{z \widetilde{R}(\varphi)}^{n}(e)+z \varphi \star\left(U_{z \widetilde{R}(\varphi)}^{n}(e) \circ Y\right) \\
& =\varphi \star\left(z \widetilde{R}(\varphi) \star U_{z \widetilde{R}(\varphi)}^{n}(e)+z U_{z \widetilde{R}(\varphi)}^{n}(e) \circ Y\right) \\
& =\varphi \star U_{z \widetilde{R}(\varphi)}^{n+1}(e) .
\end{aligned}
$$

Let us go back to the proof of Proposition 3.1. According to Lemma 3.2, we have for any $t$, at least formally,

$$
\varphi^{t}=\varphi \star \exp \left(t U_{z \widetilde{R}(\varphi)}\right)(e) .
$$

We still have to fix the convergence of the exponential just above in the case when $z \widetilde{R}(\varphi)$ belongs to $\mathcal{L}\left(\mathcal{H}, \mathcal{A}_{+}\right)$. Let us consider the following decreasing bifiltration of $\mathcal{L}\left(\mathcal{H}, \mathcal{A}_{+}\right)$:

$$
\mathcal{L}_{+}^{p, q}=\left(z^{q} \mathcal{L}\left(\mathcal{H}, \mathcal{A}_{+}\right)\right) \cap \mathcal{L}^{p}
$$

where $\mathcal{L}^{p}$ is the set of those $\alpha \in \mathcal{L}(\mathcal{H}, \mathcal{A})$ such that $\alpha(x)=0$ for any $x \in \mathcal{H}$ of degree $\leq p-1$. In particular $\mathcal{L}^{1}=\mathfrak{g}_{0}$. Considering the associated filtration

$$
\mathcal{L}_{+}^{n}=\sum_{p+q=n} \mathcal{L}_{+}^{p, q}
$$

we see that for any $\gamma \in \mathfrak{g}_{0} \cap \mathcal{L}\left(\mathcal{H}, \mathcal{A}_{+}\right)$, the transformation $U_{\gamma}$ increases the filtration by 1 , i.e.,

$$
U_{\gamma}\left(\mathcal{L}_{+}^{n}\right) \subset \mathcal{L}_{+}^{n+1}
$$

The algebra $\mathcal{L}\left(\mathcal{H}, \mathcal{A}_{+}\right)$is not complete with respect to the topology induced by this filtration, but the completion is $\mathcal{L}\left(\mathcal{H}, \widehat{\mathcal{A}_{+}}\right)$, where $\widehat{\mathcal{A}_{+}}=\mathbb{C}[[z]]$ stands for the formal series. Hence, the right-hand side of (3.9) is convergent in 
$\mathcal{L}\left(\mathcal{H}, \widehat{\mathcal{A}_{+}}\right)$with respect to this topology. Hence for any $\gamma \in \mathcal{L}\left(\mathcal{H}, \mathcal{A}_{+}\right)$and for $\varphi$ such that $z \widetilde{R}(\varphi)=\gamma$, we have $\varphi^{t}=\varphi \star h_{t}$ with $h_{t} \in \mathcal{L}\left(\mathcal{H}, \widehat{\mathcal{A}_{+}}\right)$for any $t$. On the other hand, we already know that $h_{t}$ takes values in meromorphic functions for each $t$. So $h_{t}$ belongs to $\mathcal{L}\left(\mathcal{H}, \mathcal{A}_{+}\right)$, which proves the first part of the second assertion. Equation (3.6) at $t=0$ reads

$$
z \widetilde{R}(\varphi)=\dot{h}(0)=\left.\frac{d}{d t}\right|_{t=0}\left(\varphi^{t}\right)_{+}
$$

For $\varphi \in G_{\mathcal{A}_{-}}^{\Phi}$, we have, thanks to the property $\varphi(\operatorname{Ker} \epsilon) \subset \mathcal{A}_{-}$:

$$
\begin{aligned}
h_{t}(x)=\left(\varphi^{t}\right)_{+}(x) & =(I-\pi)\left(\varphi^{t}(x)+\sum_{(x)} \varphi^{\star-1}\left(x^{\prime}\right) \varphi^{t}\left(x^{\prime \prime}\right)\right) \\
& =t(I-\pi)\left(z|x| \varphi(x)+z \sum_{(x)} \varphi^{\star-1}\left(x^{\prime}\right) \varphi\left(x^{\prime \prime}\right)\left|x^{\prime \prime}\right|\right)+O\left(t^{2}\right) \\
& =t \operatorname{Res}(\varphi \circ Y)+O\left(t^{2}\right),
\end{aligned}
$$

hence,

$$
\dot{h}(0)=\operatorname{Res}(\varphi \circ Y) .
$$

From equations (3.3), (3.4) and (3.11), we get

$$
z \widetilde{R}(\varphi)=\operatorname{Res}(\varphi \circ Y)
$$

for any $\varphi \in G_{\mathcal{A}_{-}}^{\Phi}$, hence $z \widetilde{R}(\varphi) \in \mathfrak{g}^{c}$. Conversely, let $\beta$ in $\mathfrak{g}^{c}$. Consider $\psi=$ $\widetilde{R}^{-1}\left(z^{-1} \beta\right)$. This element of $G_{\mathcal{A}}$ verifies by definition, thanks to equation (3.4):

$$
z \psi \circ Y=\psi \star \beta
$$

Hence for any $x \in \operatorname{Ker} \epsilon$, we have

$$
z \psi(x)=\frac{1}{|x|}\left(\beta(x)+\sum_{(x)} \psi\left(x^{\prime}\right) \beta\left(x^{\prime \prime}\right)\right) .
$$

As $\beta(x)$ is a constant (as a function of the complex variable $z$ ), it is easily seen by induction on $|x|$ that the right-hand side evaluated at $z$ has a limit 
when $z$ tends to infinity. Thus $\psi(x) \in \mathcal{A}_{-}$, and then

$$
\psi=\widetilde{R}^{-1}\left(\frac{1}{z} \beta\right) \in G_{\mathcal{A}_{-}}^{\Phi}
$$

which proves assertion (2).

Let us prove assertion (3): Equation $\varphi^{t}=\varphi \star h_{t}$ together with $\left(\varphi^{t}\right)^{s}=$ $\varphi^{t+s}$ yields

$$
h_{s+t}=h_{s} \star\left(h_{t}\right)^{s} .
$$

Taking values at $z=0$ immediately yields the one-parameter group property:

$$
F_{s+t}=F_{s} \star F_{t}
$$

thanks to the fact that the evaluation at $z=0$ is an algebra morphism.

We can now give a definition of the beta-function.

Definition 3.3. For any $\varphi \in G_{\mathcal{A}}^{\Phi}$, the beta-function of $\varphi$ is the generator of the one-parameter group $F_{t}$ defined by equation (3.7) in Proposition 3.1. It is the element of the dual $\mathcal{H}^{\star}$ defined by

$$
\beta(\varphi)=\left.\frac{d}{d t}\right|_{t=0} F_{t}(x)
$$

for any $x \in \mathcal{H}$.

Proposition 3.4. For any $\varphi \in G_{\mathcal{A}}^{\Phi}$, the beta-function of $\varphi$ coincides with the one of the negative part $\varphi_{-}^{\star-1}$ in the Birkhoff decomposition. It is given by any of the three expressions:

$$
\begin{aligned}
\beta(\varphi) & =\operatorname{Res} \widetilde{R}(\varphi) \\
& =\operatorname{Res}\left(\varphi_{-}^{\star-1} \circ Y\right) \\
& =-\operatorname{Res}\left(\varphi_{-} \circ Y\right) .
\end{aligned}
$$

Proof. The third equality will be derived from the second by taking residues on both sides of the equation:

$$
0=\widetilde{R}(e)=\widetilde{R}\left(\varphi_{-}\right)+\varphi_{-}^{\star-1} \star \widetilde{R}\left(\varphi_{-}^{\star-1}\right) \star \varphi_{-},
$$

which is a special instance of the cocycle formula (3.5). Suppose first $\varphi \in$ $G_{\mathcal{A}_{-}}^{\Phi}$, hence $\varphi_{-}^{\star-1}=\varphi$. Then $z \widetilde{R}(\varphi)$ is a constant according to assertion 2 of 
Proposition 3.1. The proposition then follows from equation (3.11) evaluated at $z=0$ and equation (3.12). Suppose now $\varphi \in G_{\mathcal{A}}^{\Phi}$, and consider its Birkhoff decomposition. As both components belong to $G_{\mathcal{A}}^{\Phi}$, we apply Proposition 3.1 to them. In particular, we have

$$
\begin{aligned}
\varphi^{t} & =\varphi \star h_{t}, \\
\left(\varphi_{-}^{\star-1}\right)^{t} & =\varphi_{-}^{\star-1} \star v_{t}, \\
\left(\varphi_{+}\right)^{t} & =\varphi_{+} \star w_{t},
\end{aligned}
$$

and equality $\varphi^{t}=\left(\varphi_{-}^{\star-1}\right)^{t} \star\left(\varphi_{+}\right)^{t}$ yields

$$
h_{t}=\left(\varphi_{+}\right)^{\star-1} \star v_{t} \star \varphi_{+} \star w_{t} .
$$

We denote by $F_{t}, V_{t}, W_{t}$ the one-parameter groups obtained from $h_{t}, v_{t}, w_{t}$, respectively, by letting the complex variable $z$ go to zero. It is clear that $\left.\varphi_{+}\right|_{z=0}=\mathrm{e}$, and similarly that $W_{t}$ is the constant one-parameter group reduced to the co-unit $\varepsilon$. Hence, equation (3.15) at $z=0$ reduces to

$$
F_{t}=V_{t}
$$

hence the first assertion. The cocycle equation (3.5) applied to the Birkhoff decomposition reads

$$
\widetilde{R}(\varphi)=\widetilde{R}\left(\varphi_{+}\right)+\left(\varphi_{+}\right)^{\star-1} \star \widetilde{R}\left(\varphi_{-}^{\star-1}\right) \star \varphi_{+} .
$$

Taking residues of both sides yields

$$
\operatorname{Res} \widetilde{R}(\varphi)=\operatorname{Res} \widetilde{R}\left(\varphi_{-}^{\star-1}\right),
$$

which ends the proof.

Definition 3.5. The one-parameter group $F_{t}=V_{t}$ above is the renormalization group of $\varphi[11]$.

Remark 3.6. It is possible to reconstruct $\varphi_{-}$from $\beta(\varphi)$ using a scatteringtype formula $[11,8,15,39]$. Hence $\varphi_{-}$(i.e., the divergence structure of $\varphi$ ) is uniquely determined by its residue.

\subsection{The matrix representation of the grading derivation}

As in reference [11], denote by $Z_{0}$ the derivation of the algebra $\mathcal{L}(\mathcal{H}, \mathcal{A})$ (which is also a derivation of the Lie algebra $\mathfrak{g}_{\mathcal{A}}$ ) given by $\alpha \mapsto \alpha \circ Y$. Let $\widetilde{\mathcal{L}}$ 
be the semi-direct product of $\mathcal{L}(\mathcal{H}, \mathcal{A})$ with $Z_{0}$, and let $\widetilde{\mathfrak{g}_{\mathcal{A}}}$ be the semi-direct product $\mathfrak{g}_{\mathcal{A}} \rtimes \mathbb{C} . Z_{0}$. Similarly, let $\widetilde{G}_{\mathcal{A}}=G_{\mathcal{A}} \rtimes \mathbb{C}$ be the semi-direct product of the group $G_{\mathcal{A}}$ by the one-parameter group $\theta_{t}=\exp t Z_{0}$ of automorphisms. Let $J$ be any graded left coideal of $\mathcal{H}$. We suppose further that the filtrationordered basis $\left(x_{i}\right)_{i \in I}$ of $J$ is graded, i.e., made of homogeneous elements. The degree of $x_{i}$ will be denoted by $\left|x_{i}\right|$. We want to extend the matrix representation $\Psi_{J}$ from $\mathcal{L}(\mathcal{H}, \mathcal{A})$ to $\widetilde{\mathcal{L}}$. This can be done by representing $Z_{0}$ by a diagonal matrix.

Proposition 3.7. The correspondence $\Psi_{J}: \widetilde{\mathcal{L}} \rightarrow \operatorname{End}_{\mathcal{A}}(\mathcal{A} \otimes J)$ defined as in Paragraph 2.3 on $\mathcal{L}(\mathcal{H}, \mathcal{A})$, and such that

$$
\Psi_{J}\left[Z_{0}\right]\left(x_{i}\right)=\left|x_{i}\right| \cdot x_{i}
$$

is an algebra morphism.

Proof. We only have to show the equality: ${ }^{3}$

$$
\left[\Psi_{J}[f], \Psi_{J}\left[-Z_{0}\right]\right]=\Psi_{J}\left(\left[f,-Z_{0}\right]\right)=\Psi_{J}(f \circ Y) .
$$

This follows by a direct computation

$$
\begin{aligned}
{\left[\Psi_{J}[f], \Psi_{J}\left[-Z_{0}\right]\right]\left(x_{j}\right) } & =-\Psi_{J}[f] \Psi_{J}\left[Z_{0}\right]\left(x_{j}\right)+\Psi_{J}\left[Z_{0}\right] \Psi_{J}[f]\left(x_{j}\right) \\
& =\sum_{i}\left(\left|x_{i}\right|-\left|x_{j}\right|\right) f\left(M_{i j}\right) \otimes x_{i},
\end{aligned}
$$

whereas

$$
\begin{aligned}
\Psi_{J}[f \circ Y]\left(x_{j}\right) & =\sum_{i}(f \circ Y)\left(M_{i j}\right) \otimes x_{i} \\
& =\sum_{i}\left|M_{i j}\right| f\left(M_{i j}\right) \otimes x_{i} .
\end{aligned}
$$

By definition of the coproduct matrix, and thanks to the fact that $\mathcal{H}$ is graded, the coefficients $M_{i j}$ are homogeneous of degree $\left|x_{i}\right|-\left|x_{j}\right|$, which finishes the proof.

\footnotetext{
${ }^{3}$ There is a minus sign in front of $Z_{0}$ due to the fact that we have put it on the right inside of the bracket, reflecting the fact that the action of the one-parameter group has been written on the right.
} 
By taking exponentials of the above diagonal matrices, we of course get a matrix representation of the one-parameter group $\theta_{t}(3.1)$, namely

$$
\Psi_{J}\left[\exp t Z_{0}\right]\left(x_{i}\right)=\mathrm{e}^{t\left|x_{i}\right|} x_{i}
$$

\subsection{Matrix differential equations}

We fix a graded coideal $J$ of $\mathcal{H}$, and we therefore introduce the following notation:

$$
\widehat{f}:=\Psi_{J}[f]
$$

Keeping the notations of Paragraph 3.1, we have then, as a consequence of Propositions 2.4 and 3.7:

$$
\widehat{\varphi^{t}}(z)=\mathrm{e}^{t z \widehat{Z_{0}}} \widehat{\varphi}(z) \mathrm{e}^{-t z \widehat{Z_{0}}}
$$

as an equality of size $|I|$ square matrices with coefficients in meromorphic functions of the complex variable $z$. With respect to the example left coideal generated by $\mathcal{T}^{\prime} \subset \mathcal{T}$ in (2.20), we find

$$
\widehat{Z_{0}}=\left(\begin{array}{ccccc}
0 & 0 & 0 & 0 & 0 \\
0 & 1 & 0 & 0 & 0 \\
0 & 0 & 2 & 0 & 0 \\
0 & 0 & 0 & 3 & 0 \\
0 & 0 & 0 & 0 & 4
\end{array}\right) \quad \text { and } \quad \mathrm{e}^{t z \widehat{Z_{0}}}=\left(\begin{array}{ccccc}
1_{\mathcal{A}} & 0 & 0 & 0 & 0 \\
0 & \mathrm{e}^{z t} & 0 & 0 & 0 \\
0 & 0 & \mathrm{e}^{2 z t} & 0 & 0 \\
0 & 0 & 0 & \mathrm{e}^{3 z t} & 0 \\
0 & 0 & 0 & 0 & \mathrm{e}^{4 z t}
\end{array}\right)
$$

We now change the general notations slightly and consider $\widehat{\varphi}$ as a function of the variable $t \in \mathbb{C}$ with values in $\mathcal{A} \otimes J$ (i.e., as a matrix-valued function of both variables $(t, z))$. More precisely, we put

$$
\widehat{\varphi}(t, z)=\mathrm{e}^{t z \widehat{Z_{0}}} \widehat{\varphi}(0, z) \mathrm{e}^{-t z \widehat{Z_{0}}},
$$

where $\widehat{\varphi}(0, z)$ stands for the old $\widehat{\varphi}(z)$. Now $\widehat{\varphi}_{-}(t)$ (resp. $\left.\widehat{\varphi}_{+}(t)\right)$ will stand for the negative (resp. positive) component of the Birkhoff decomposition of $\widehat{\varphi}(t)(2.31)$, for any $t \in \mathbb{C}$. Using the toy-model character $\varphi=\varphi(s, z)$ in 
equation (2.13) with parameterized 't Hooft mass, we find explicitly

$$
\begin{aligned}
& \widehat{\varphi}^{t}(z)=\widehat{\varphi}(t, z) \\
& =\left(\begin{array}{ccccc}
1_{\mathcal{A}} & 0 & 0 & 0 & 0 \\
\frac{B_{1}}{\left(\mathrm{e}^{-t} \alpha(0)\right)^{z}} & 1_{\mathcal{A}} & 0 & 0 & 0 \\
\frac{B_{2} B_{1}}{\left(\mathrm{e}^{-t} \alpha(0)\right)^{2 z}} & \frac{B_{1}}{\left(\mathrm{e}^{-t} \alpha(0)\right)^{z}} & 1_{\mathcal{A}} & 0 & 0 \\
\frac{B_{3} B_{1}^{2}}{\left(\mathrm{e}^{-t} \alpha(0)\right)^{3 z}} & \frac{B_{1}^{2}}{\left(\mathrm{e}^{-t} \alpha(0)\right)^{2 z}} & 2 \frac{B_{1}}{\left(\mathrm{e}^{-t} \alpha(0)\right)^{z}} & 1_{\mathcal{A}} & 0 \\
\frac{B_{4} B_{1}^{3}}{\left(\mathrm{e}^{-t} \alpha(0)\right)^{4 z}} & \frac{B_{1}^{3}}{\left(\mathrm{e}^{-t} \alpha(0)\right)^{3 z}} & 3 \frac{B_{1}^{2}}{\left(\mathrm{e}^{-t} \alpha(0)\right)^{2 z}} & 3 \frac{B_{1}}{\left(\mathrm{e}^{-t} \alpha(0)\right)^{z}} & 1_{\mathcal{A}}
\end{array}\right) \\
& =\mathrm{e}^{t z \widehat{Z_{0}}}\left(\begin{array}{ccccc}
1_{\mathcal{A}} & 0 & 0 & 0 & 0 \\
\frac{B_{1}}{\alpha(0)^{z}} & 1_{\mathcal{A}} & 0 & 0 & 0 \\
\frac{B_{2} B_{1}}{\alpha(0)^{2 z}} & \frac{B_{1}}{\alpha(0)^{z}} & 1_{\mathcal{A}} & 0 & 0 \\
\frac{B_{3} B_{1}^{2}}{\alpha(0)^{3 z}} & \frac{B_{1}^{2}}{\alpha(0)^{2 z}} & 2 \frac{B_{1}}{\alpha(0)^{z}} & 1_{\mathcal{A}} & 0 \\
\frac{B_{4} B_{1}^{3}}{\alpha(0)^{4 z}} & \frac{B_{1}^{3}}{\alpha(0)^{3 z}} & 3 \frac{B_{1}^{2}}{\alpha(0)^{2 z}} & 3 \frac{B_{1}}{\alpha(0)^{z}} & 1_{\mathcal{A}}
\end{array}\right) \mathrm{e}^{-t z \widehat{Z_{0}}} .
\end{aligned}
$$

Hence, we observe the simple transformation on $\widehat{\varphi}(z)=\widehat{\varphi}(t, z)$, where $t$ parameterizes 't Hooft's unit mass (2.14), i.e., $\alpha(t):=a / \mathrm{e}^{t} \mu=\mathrm{e}^{-t} \alpha(0)>0$ :

$$
\widehat{\varphi}^{0}(z):=\widehat{\varphi}(0, z) \stackrel{A d\left[\mathrm{e}^{t z \widehat{Z_{0}}}\right]}{\longrightarrow} \widehat{\varphi}^{t}(z)=\widehat{\varphi}(t, z) .
$$

We introduce the auxiliary matrix

$$
A:=\widehat{\varphi} \mathrm{e}^{t z \widehat{Z_{0}}},
$$

as well as its Birkhoff decomposition

$$
A=A_{-}^{-1} A_{+} \text {, with } A_{-}=\widehat{\varphi}_{-} \text {and } A_{+}=\widehat{\varphi}_{+} \mathrm{e}^{t z \widehat{Z_{0}}} .
$$

From the obvious equality

$$
A(t)=\mathrm{e}^{t z \widehat{Z_{0}}} A \mathrm{e}^{-t z \widehat{Z_{0}}}=\mathrm{e}^{t z \widehat{Z_{0}}} A(0),
$$

we get by differentiating with respect to $t$ :

$$
\frac{d}{d t} A=\dot{A}=z \widehat{Z_{0}} A \text {. }
$$


Using the Birkhoff decomposition of $A$ then yields

$$
z \widehat{Z_{0}} \widehat{\varphi}_{-}^{-1} A_{+}=\dot{\hat{\varphi}}_{-}^{-1} A_{+}+\widehat{\varphi}_{-}^{-1} \dot{A}_{+} .
$$

Multiplying both sides with $\widehat{\varphi}_{-}$on the left and with $A_{+}^{-1}$ on the right, we get

$$
\widehat{\varphi}_{-}\left(z{\widehat{Z_{0}}}\right) \widehat{\varphi}_{-}^{-1}=\widehat{\varphi}_{-} \dot{\hat{\varphi}}_{-}^{-1}+\dot{A}_{+} A_{+}^{-1} .
$$

Suppose now that $\varphi(0)$ belongs to $G_{\mathcal{A}}^{\Phi}$, which implies for the corresponding matrix $\widehat{\varphi}(0)=\widehat{\varphi}_{-}^{-1} \widehat{\varphi}_{+}$:

$$
\frac{d}{d t} \widehat{\varphi}_{-}=\dot{\hat{\varphi}}_{-}=0
$$

Then equation (3.28) reduces to:

$$
\widehat{\varphi}_{-}\left(z \widehat{Z}_{0}\right) \widehat{\varphi}_{-}^{-1}=\dot{A}_{+} A_{+}^{-1} .
$$

\subsection{The renormalization group and the beta-function in the matrix setting}

Keeping the notations of the previous paragraph, it is clear that if $\varphi(0)$ belongs to $G_{\mathcal{A}}^{\Phi}$, then $\varphi(t) \in G_{\mathcal{A}}^{\Phi}$ for any $t$, and moreover the renormalization group and the beta-function of $\varphi(t)$ do not depend on $t$. We can then talk about the renormalization group and the beta-function of $\varphi$ without mentioning a particular value of $t$.

Theorem 3.8. The matrix representation of the beta-function reads

$$
\widehat{\beta}(\varphi)=\widehat{\varphi}_{-}\left(z \widehat{Z_{0}}\right) \widehat{\varphi}_{-}^{-1}-z \widehat{Z_{0}} .
$$

Proof. This is a direct computation of (scalar-valued) matrices, see equation (3.16) and Definition 3.5

$$
\widehat{F}_{t}=\lim _{z \rightarrow 0}\left(\widehat{\varphi}_{-}(z) \mathrm{e}^{z t \widehat{Z}_{0}} \widehat{\varphi}_{-}^{-1}(z) \mathrm{e}^{-z t \widehat{Z}_{0}}\right) .
$$

The limit exists by Proposition 3.1. The term inside the bracket on the righthand side is holomorphic at zero as a function of $z$, and so is its derivative with respect to $t$. The operation $\frac{\partial}{\partial t}$ commutes then with evaluating at $z=0$, and we get by definition of the beta-function:

$$
\begin{aligned}
\widehat{\beta}(\varphi) & =\left.\lim _{z \rightarrow 0} \frac{\partial}{\partial t}\left(\widehat{\varphi}_{-}(z) \mathrm{e}^{z t \widehat{Z}_{0}} \widehat{\varphi}_{-}^{-1}(z) \mathrm{e}^{-z t \widehat{Z_{0}}}\right)\right|_{t=0} \\
& =\lim _{z \rightarrow 0}\left(\widehat{\varphi}-(z) z \widehat{Z_{0}} \widehat{\varphi}_{-}^{-1}(z)-z \widehat{Z_{0}}\right) .
\end{aligned}
$$

Now, subtracting $z \widehat{Z_{0}}$ on both sides of equation (3.30) gives an expression on the left-hand side which admits a limit when $z \rightarrow \infty$ and a term on the 
right-hand side which admits a limit when $z \rightarrow 0$. Hence, the term

$$
\widehat{\varphi}_{-}(z) z{\widehat{Z_{0}}}_{0}^{-1}(z)-z{\widehat{Z_{0}}}_{-}=\widehat{\varphi}_{-}(z)\left[\widehat{\varphi}_{-}^{-1}(z),-z \widehat{Z}_{0}\right]
$$

is a matrix with constant coefficients, which proves the theorem.

Coming back to Remark 2.8, we find immediately for the matrix betafunction the simple equation in the Lie algebra $\widehat{\mathfrak{g}}_{\mathcal{A}}$, in accordance with results in [39]:

$$
\begin{aligned}
\widehat{\beta}(\varphi)= & \exp (\mathrm{R}(\chi(\widehat{Z})))\left(z \widehat{Z_{0}}\right) \exp (-\mathrm{R}(\chi(\widehat{Z})))-z \widehat{Z_{0}} \\
= & z\left[\mathrm{R}(\chi(\widehat{Z})), \widehat{Z_{0}}\right]+\frac{z}{2 !}\left[\mathrm{R}(\chi(\widehat{Z})),\left[\mathrm{R}(\chi(\widehat{Z})), \widehat{Z_{0}}\right]\right] \\
& +\frac{z}{3 !}\left[\mathrm{R}(\chi(\widehat{Z})),\left[\mathrm{R}(\chi(\widehat{Z})),\left[\mathrm{R}(\chi(\widehat{Z})), \widehat{Z_{0}}\right]\right]\right]+\cdots \\
= & z \sum_{n>0} \frac{1}{n !} \operatorname{ad}[\mathrm{R}(\chi(\widehat{Z}))]^{(n)}\left(\widehat{Z_{0}}\right) .
\end{aligned}
$$

The following corollary is a direct consequence of equation (3.30).

\section{Corollary 3.9.}

$$
\dot{A}_{+} A_{+}^{-1}=\widehat{\beta}(\varphi)+z \widehat{Z_{0}} .
$$

and for the renormalization matrix, we get

\section{Corollary 3.10.}

$$
\widehat{\varphi}_{+}(t, z)=\mathrm{e}^{t\left(\widehat{\beta}(\varphi)+z \widehat{Z_{0}}\right)} \widehat{\varphi}_{+}(z, 0) \mathrm{e}^{-t z \widehat{Z_{0}}} .
$$

Proof. From Corollary 3.9, we get

$$
A_{+}(t)=\mathrm{e}^{t\left(\widehat{\beta}(\varphi)+z \widehat{Z_{0}}\right)} A_{+}(0),
$$

which immediately proves the claim. Alternatively, one readily observes that

$$
\begin{aligned}
\widehat{\varphi}_{+}(t, z)=\widehat{\varphi}-\widehat{\varphi} t & =\widehat{\varphi}_{-} \mathrm{e}^{t z \widehat{Z_{0}}} \widehat{\varphi}(0, z) \mathrm{e}^{-t z \widehat{Z_{0}}} \\
& =\widehat{\varphi}_{-} \mathrm{e}^{t z \widehat{Z_{0}}} \widehat{\varphi}_{-}^{-1} \widehat{\varphi}_{+}(0, z) \mathrm{e}^{-t z \widehat{Z_{0}}} \\
& =\mathrm{e}^{t \widehat{\varphi}_{-}\left(z \widehat{Z_{0}}\right) \widehat{\varphi}_{-}^{-1}} \widehat{\varphi}_{+}(z, 0) \mathrm{e}^{-t z \widehat{Z_{0}}} \\
& =\mathrm{e}^{t\left(\widehat{\beta}(\varphi)+z \widehat{Z_{0}}\right)} \widehat{\varphi}_{+}(z, 0) \mathrm{e}^{-t z \widehat{Z_{0}}},
\end{aligned}
$$

where we used the well-known fact

$$
\exp (A) \exp (B) \exp (-A)=\exp (\exp (A) B \exp (-A)) .
$$


Corollary 3.11. (Connes-Kreimer's scattering-type formula [9]).

$$
\widehat{\varphi}_{-}=\lim _{t \rightarrow+\infty} \mathrm{e}^{-t\left(\widehat{\beta}(\varphi) / z+\widehat{Z_{0}}\right)} \mathrm{e}^{t \widehat{Z_{0}}} .
$$

Proof. We again adapt Sakakibara's computation [[43], § 2] to our matrix setting:

$$
\begin{aligned}
\mathrm{e}^{-t\left(\widehat{\beta}(\varphi) / z+\widehat{Z_{0}}\right)} \mathrm{e}^{t \widehat{Z_{0}}} & =\mathrm{e}^{-t\left(\widehat{\varphi}-\widehat{Z}_{0} \widehat{\varphi}_{-}^{-1}\right)} \mathrm{e}^{t \widehat{Z_{0}}} \\
& =\widehat{\varphi}_{-} \mathrm{e}^{-t \widehat{Z}_{0}} \widehat{\varphi}_{-}^{-1} \mathrm{e}^{t \widehat{Z_{0}}} \\
& =\Psi_{J}\left[\varphi_{-} \star \theta_{-t}\left(\varphi_{-}^{\star-1}\right)\right] .
\end{aligned}
$$

Now we have for any homogeneous $x \in \mathcal{H}$ of degree $\geq 1$,

$$
\begin{aligned}
\lim _{t \rightarrow+\infty}\left(\varphi_{-} \star \theta_{-t}\left(\varphi_{-}^{\star-1}\right)\right)(x)= & \lim _{t \rightarrow+\infty}\left(\varphi_{-}(x)+\mathrm{e}^{-t|x|} \varphi_{-}^{-1}(x)\right. \\
& \left.+\sum_{(x)} \varphi_{-}\left(x^{\prime}\right) \mathrm{e}^{-t\left|x^{\prime \prime}\right|} \varphi_{-}^{-1}\left(x^{\prime \prime}\right)\right) \\
= & \varphi_{-}(x) .
\end{aligned}
$$

Replacing $x$ with the matrix coefficients $M_{i j}$ proves then the corollary.

This result becomes evident on the level of matrices when going back to equation (3.23). Assume for a moment $z \in \mathbb{R}$ positive. One observes by replacing $t$ by $-t$ that in the first equality on the right-hand side each

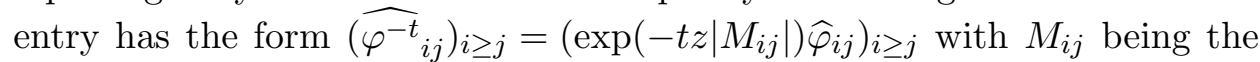
coproduct matrix in (2.21). Hence, with $\left|M_{i i}\right|=0$ we see immediately that \left.${\widehat{\left(\varphi^{-t}\right.}}_{i j}\right) \stackrel{t \rightarrow \infty}{\longrightarrow} \mathbf{1}$.

Remark 3.12. Considering Proposition 3.4 in the matrix setting, we have an alternative matrix representation of the beta-function:

$$
\widehat{\beta}(\varphi)=\left[\operatorname{Res} \widehat{\varphi}_{-}, \widehat{Z}_{0}\right]
$$

\section{Acknowledgments}

The first author acknowledges greatly the support by the European PostDoctoral Institute and Institut des Hautes Études Scientifiques (I.H.É.S.). He profited from discussions with D. Kreimer. The second author greatly acknowledges constant support from the Centre National de la Recherche Scientifique (C.N.R.S.). 


\section{References}

[1] E. Abe, Hopf algebras, Cambridge University Press, Cambridge, 1980.

[2] F.V. Atkinson, Some aspects of Baxter's functional equation, J. Math. Anal. Appl. 7 (1963) 1.

[3] G.M. Bergman, Everybody knows what a Hopf algebra is, Group actions on rings (Brunswick, Maine, 1984), Contemp. Math. 43 (1985), 25.

[4] D.J. Broadhurst and D. Kreimer, Renormalization automated by Hopf algebra, J. Symb. Comput. 27 (1999), 581.

[5] L. Brown (ed.), Renormalization. From Lorentz to Landau (and beyond), Springer-Verlag, New York, 1993.

[6] W.E. Caswell and A.D. Kennedy, A simple approach to renormalization theory, Phys. Rev. D 25 (1982) 392.

[7] V. Chari and A. Pressley, A guide to Quantum Groups, Cambridge Univeristy Press, 1995.

[8] A. Connes, Symétries galoisiennes et renormalization, Séminaire Poincaré 2 (2002), 75.

[9] A. Connes and D. Kreimer, Hopf algebras, renormalization and noncommutative geometry, Commun. Math. Phys. 199 (1998), 203.

[10] A. Connes and D. Kreimer, Renormalization in quantum field theory and the Riemann-Hilbert problem. I. The Hopf algebra structure of graphs and the main theorem, Commun. Math. Phys. 210 (2000), 249.

[11] A. Connes and D. Kreimer, Renormalization in quantum field theory and the Riemann-Hilbert problem. II. The $\beta$-function, diffeomorphisms and the renormalization group, Commun. in Math. Phys. 216 (2001), 215.

[12] A. Connes and D. Kreimer, Insertion and elimination: the doubly infinite Lie algebra of Feynman graphs, Ann. Inst. Henri Poincaré 3 (2002), 411.

[13] J.C. Collins, Renormalization, Cambridge Monographs on Mathematical Physics, Cambridge University Press, Cambridge, 1984.

[14] J.C. Collins, Renormalization: general theory, contribution to the Encyclopedia of Mathematical Physics, Elsevier, hep-th/0602121.

[15] A. Connes and M. Marcolli, From Physics to number theory via noncommutative geometry, Part II: renormalization, the Riemann-Hilbert correspondence, and motivic Galois theory, to appear in the volume 'Frontiers in Number Theory, Physics, and Geometry' hep-th/0411114.

[16] B. Delamotte, A hint of renormalization, Am. J. Phys. 72 (2004), 170 . 
[17] K. Ebrahimi-Fard, L. Guo and D. Kreimer, Spitzer's identity and the algebraic Birkhoff decomposition in pQFT, J. Phys. A Math. Gen. 37 (2004), 11037.

[18] K. Ebrahimi-Fard, L. Guo and D. Kreimer, Integrable renormalization II: the general case, Ann. H. Poincaré 6 (2005), 369.

[19] K. Ebrahimi-Fard, J.M. Gracia-Bondía, L. Guo and J.C. Várilly, Combinatorics of renormalization as matrix calculus, Phys. Lett. B. 632 (2006), 552 .

[20] K. Ebrahimi-Fard and L. Guo, Matrix representation of renormalization in perturbative quantum field theory, hep-th/0508155.

[21] K. Ebrahimi-Fard and L. Guo, Rota-Baxter Algebras in Renormalization of Perturbative Quantum Field Theory, Fields Institute Communications 50 (2007), 47.

[22] K. Ebrahimi-Fard and D. Kreimer, Hopf algebra approach to Feynman diagram calculations, J. Phys. A Math. Gen. 38 (2005), R385.

[23] K. Ebrahimi-Fard, L. Guo and D. Manchon, Birkhoff type decompositions and the Baker-Campbell-Hausdorff recursion, Commun. Math. Phys. 267 (2006), 821.

[24] H. Figueroa and J.M. Gracia-Bondía, On the antipode of Kreimer's Hopf algebra, Mod. Phys. Lett. A16 (2001), 1427.

[25] H. Figueroa and J.M. Gracia-Bondía, Combinatorial Hopf algebras in quantum field theory I, Rev. Math. Phys. 17 (2005), 881.

[26] H. Figueroa, J.M. Gracia-Bondía and J.C. Várilly, Elements of noncommutative geometry, Birkhäuser, 2001.

[27] C. Itzykson and J.-B. Zuber, Quantum field theory, McGraw-Hill, NewYork, 1980.

[28] S.A. Joni and G.-C. Rota, Coalgebras and bialgebras in combinatorics, Stud. Appl. Math. 61 (1979), 93. Reprinted in: 'Gian-Carlo Rota on combinatorics: Introductory papers and commentaries', ed. J.P.S. Kung, Contemp. Mathematicians, Birkhäuser Boston, Boston, MA, 1995.

[29] D. Kaiser, Drawing theories apart: the dispersion of Feynman diagrams in postwar physics, The University of Chicago Press, Chicago, 2005.

[30] C. Kassel, Quantum groups, Springer-Verlag, New York, 1995.

[31] D. Kreimer, On the Hopf algebra structure of perturbative quantum field theories, Adv. Theor. Math. Phys. 2 (1998), 303.

[32] D. Kreimer, Chen's iterated integral represents the operator product expansion, Adv. Theor. Math. Phys. 3 (1999), 627. 
[33] D. Kreimer, On overlapping divergencies, Commun. Math. Phys. 204 (1999), 669.

[34] D. Kreimer, Knots and Feynman diagrams, Cambridge Monographs on Mathematical Physics, Cambridge University Press, Cambridge, 2000.

[35] D. Kreimer, Combinatorics of (perturbative) quantum field theory, Phys. Rep. 363 (2002), 387.

[36] D. Kreimer, Factorization in quantum field theory: an exercise in Hopf algebras and local singularities, contributed to Les Houches School of Physics: 'Frontiers in Number Theory, Physics and Geometry', Les Houches, France, 9-21, March 2003, hep-th/0306020.

[37] D. Kreimer and R. Delbourgo, Using the Hopf algebra structure of QFT in calculations, Phys. Rev. D, 60 (1999) 105025.

[38] S. Majid, Foundations of quantum group theory, Cambridge University Press, Cambridge, 1995.

[39] D. Manchon, Hopf algebras, from basics to applications to renormalization, Comptes-rendus des Rencontres mathématiques de Glanon, 2001.

[40] T. Muta, Foundations of quantum chromodynamics: an introduction to perturbative methods in gauge theories, World Sci. Lect. Notes Phys. 5 (1987).

[41] W. Nichols and M. Sweedler, Hopf algebras and combinatorics. Umbral calculus and Hopf algebras (Norman, Okla., 1978), Contemp. Math. 6 (1982), 49.

[42] G.-C. Rota, Hopf algebras in combinatorics, in 'Hopf algebra methods in combinatorics. Problèmes combinatoires et théorie des graphes', (Colloq. Internat. CNRS, Univ. Orsay, Orsay, 1976), 363-365, Colloq. Internat. CNRS, 260, CNRS, Paris, 1978. Reprinted in: 'Gian-Carlo Rota on Combinatorics: Introductory papers and commentaries', ed. J.P.S. Kung, Contemp. Mathematicians, Birkhäuser Boston, Boston, MA, 1995.

[43] M. Sakakibara, On the differential equations of the characters for the renormalization group, Mod. Phys. Lett. A 19 (2004), 1453.

[44] S. Schweber, QED and the men who made it: Dyson, Feynman, Schwinger and Tomonoga, Princeton University Press, Princeton, 1994.

[45] J. Schwinger (ed.), Selected papers on quantum electrodynamics, Dover, New York, 1958.

[46] W. Schmitt, Hopf algebras in enumerative combinatorics, in 'GianCarlo Rota on combinatorics, Introductory papers and commentaries', ed. J.P.S. Kung, Contemp. Mathematicians, Birkhäuser Boston, Boston, MA, 1995. 
[47] E. Spiegel and C.J. O'Donnell, Incidence algebras, Vol. 206, Dekker Incorporated, Marcel, 1997.

[48] S. Shnider and S. Sternberg, Quantum groups. From coalgebras to Drinfel'd algebras. A guided tour, Graduate Texts in Mathematical Physics, II. International Press, Cambridge, MA, 1993.

[49] M.E. Sweedler, Hopf algebras, Benjamin, New-York, 1969.

[50] G. 't Hooft and M.J.G. Veltman, Diagrammar, Louvain 1973, Particle interactions at very high energies, Part B*, New York 1973, 177-322 and CERN Geneva-CERN 73-9 (73,REC.OCT).

[51] A.N. Vasilev, The field theoretic renormalization group in critical behavior theory and stochastic dynamics, Chapman \& Hall/CRC, Boca Raton, FL, 2004. 